\title{
Ground Moving Target Imaging Using Ultranarrowband Continuous Wave Synthetic Aperture Radar
}

\author{
Ling Wang, Member, IEEE, and Birsen Yazici, Senior Member, IEEE
}

\begin{abstract}
We present a novel method for ground moving target imaging using a synthetic aperture radar system transmitting ultranarrowband continuous waveforms $(\mathrm{CW})$. Our method exploits the high Doppler resolution provided by ultranarrowband CW signals to image both the scene reflectivity and to determine the velocity of multiple moving targets. We develop a new forward model based on the temporal Doppler induced by the movement of antennas and moving targets. The forward model relates reflectivity and velocity information at each location to a correlated received signal. We form the reflectivity images of the moving targets and estimate their motion parameters using a filtered-backprojection (FBP) technique combined with the contrast or gradient optimization method. The method results in focused reflectivity images of moving targets and their velocity estimates, regardless of the target location, speed, and velocity direction. We show that the amplitude and visible edges of the targets can be correctly reconstructed when the correct target velocity estimate is used in the FBP imaging. We present the resolution analysis of the reflectivity images. Extensive numerical simulations demonstrate the performance of our method and validate the theoretical results.
\end{abstract}

Index Terms-Continuous wave (CW), doppler, filteredbackprojection, imaging, moving target, synthetic aperture.

\section{INTRODUCTION}

G ROUND moving target imaging using synthetic aperture radar (SAR) has received considerable attention in the past two decades [1]-[21]. However, all the existing techniques are for traditional SAR systems, which accomplish high-range resolution imaging of static scenes and moving targets by transmitting wideband waveforms. We consider the problem of synthetic aperture moving target imaging using ultranarrowband continuous waveforms (CWs). Such waveforms offer a number of advantages. First, a SAR system that uses such signals

Manuscript received June 28, 2012; revised June 24, 2013; accepted June 25, 2013. Date of publication July 19, 2013; date of current version August 30, 2013. This work was supported in part by the Air Force Office of Scientific Research under Agreements FA9550-09-1-0013 and FA9550-121-0415 and in part by the National Science Foundation under Grants CCF08030672 and CCF-1218805.

L. Wang is with the Department of Information and Communication Engineering, Nanjing University of Aeronautics and Astronautics, Nanjing 210016, China (e-mail: wanglrpi@gmail.com).

B. Yazici is with the Department of Electrical, Computer, and Systems Engineering, Rensselaer Polytechnic Institute, Troy, NY 12180 USA (e-mail: B. Y: yazici@ecse.rpi.edu).

Color versions of one or more of the figures in this paper are available online at http://ieeexplore.ieee.org.

Digital Object Identifier 10.1109/TGRS.2013.2272603 requires a relatively simple and low-cost transmitter and, in some cases, does not need a dedicated transmitter. Existing radio frequency signals, such as radio, television signals, WiFi signals, etc., can be used as the illumination sources. Second, ultranarrowband CWs have high Doppler resolution and are capable of capturing the velocity information of the moving targets, as compared with the high-range-resolution waveforms used by the traditional SAR systems.

In [22]-[24], we presented a novel synthetic aperture imaging method of stationary scenes that takes advantage of the high Doppler resolution of the transmitted ultranarrowband CWs. In this paper, we consider the moving target imaging problem using such an active bistatic SAR system. Our approach exploits the high Doppler resolution of the transmitted waveforms to reconstruct the reflectivity (position), as well as to estimate the velocity field of moving targets. We derive a novel forward model that includes temporal Doppler parameters induced by the movement of the antennas and moving targets and relates the velocity field and reflectivity of the scene to a correlated received signal. We develop a novel filtered-backprojection (FBP)-type image formation method combined with image contrast and gradient optimization to reconstruct the scene reflectivity and to determine the velocity of moving targets.

Our work differs significantly from the existing work in wideband pulsed SAR imaging of moving targets in many aspects. Conventional pulsed SAR moving target imaging methods rely on high range resolution and ignore the temporal Doppler since wideband pulsed waveforms have poor Doppler resolution. Furthermore, they rely on the start-stop approximation [25], [26]. Our imaging method does not make the start-stop approximation, ignore the delay measurements and, instead, uses the high-temporal Doppler measurements provided by the ultranarrowband $\mathrm{CW}$ signals. Note that frequencymodulated CW (FMCW) SAR processing takes into account the temporal Doppler in imaging [27], [28]. However, the moving target detection or imaging methods developed for FMCW SAR rely primarily on the high range resolution and do not utilize the high Doppler resolution provided by CWs.

We adopt an approach similar to [6], [10], [15], and [17] and form a set of reflectivity images for a range of hypothesized velocities for each scatterer. Unlike the traditional SAR reflectivity imaging where the measurements are backprojected onto the constant range contours, we form reflectivity images by filtering and backprojecting the preprocessed received signal onto the constant bistatic temporal Doppler contours for a given 
hypothesized velocity. The scatterers that lie on the constant bistatic Doppler contours can be determined with high resolution due to high-resolution Doppler measurements. We show that, when the hypothesized velocity is equal to the correct velocity of a scatterer at a given location, the visible edges of the scene are reconstructed at the correct location and orientation. We design the filter so that the edges are reconstructed at the correct strength whenever the hypothesized velocity field is equal to the true velocity field. This filter depends not only on the antenna beam patterns, geometric spreading factors, etc., but also on the hypothesized target velocity. We next use the image contrast and image gradient of the reflectivity images as figures of merit to measure how well the reconstructed reflectivity images are focused and, hence, to determine the velocity of moving targets. We present the point spread function (PSF) analysis and the resolution analysis of our method. The PSF analysis shows that our reflectivity image reconstruction method takes advantage of not only the temporal Doppler induced by the movement of the antennas and moving targets but also the acceleration of antennas and moving targets in certain directions. We analyze the resolution of the reconstructed reflectivity images. Our analysis identifies several factors related to the imaging geometry and the transmitted waveforms that effect the resolution of reflectivity images. We present extensive numerical simulations to demonstrate the performance of our method and to validate the theoretical findings. To the best of our knowledge, our method is the first in the literature that addresses the synthetic aperture ground moving target detection and imaging using such waveforms.

In addition to the advantages provided by the ultranarrowband CW signals, our moving target imaging method also has several other advantages over the existing methods: 1) Unlike [1], [6], [7], [10], [11], [13], [15], [20], [21], and [29]-[31], our method can reconstruct the images of multiple moving targets, regardless of the target speed, the direction of target velocity, and target location, and determine the 2-D velocity of ground moving targets. Furthermore, our method can reconstruct highresolution images of stationary and moving targets simultaneously. 2) Unlike [2]-[4], [11], [13], [29], and [30], our imaging method does not require a priori knowledge of the target motion parameters. 3) Our method focuses moving targets at the correct locations in the reconstructed reflectivity images. The techniques for the localization or reposition of moving targets used in most conventional SAR or ground moving target indicator methods are not needed [6], [10], [15], [20]. 4) Our method can be easily extended to accommodate arbitrary target motions, such as accelerating targets. 5) It is applicable to arbitrary imaging geometries, including arbitrary flight trajectories and nonflat topography. Furthermore, our image formation method is analytic and can be implemented computationally efficiently [32].

The organization of the paper is as follows: In Section II, we present the model for the received signal and develop the forward model for moving target imaging using ultranarrowband CWs. In Section III, we develop an FBP-type image formation method to reconstruct the scene reflectivity and two velocity estimation methods. In Section IV, we present the resolution analysis of the reconstructed reflectivity images. In
Section V, we present numerical simulations to demonstrate the performance of our moving target imaging method. Section VI concludes our paper.

We use the following notational conventions throughout the paper. The bold Roman, bold italic, and Roman lower-case letters are used to denote variables in $\mathbb{R}^{3}, \mathbb{R}^{2}$, and $\mathbb{R}$, respectively, i.e., $\mathbf{z}=(\boldsymbol{z}, z) \in \mathbb{R}^{3}$, with $\boldsymbol{z} \in \mathbb{R}^{2}$ and $z \in \mathbb{R}$. The calligraphic letters $(\mathcal{F}, \mathcal{K}$, etc.) are used to denote operators.

\section{FORWARD MODEL FOR MOVING TARGET IMAGING}

\section{A. Model for Moving Targets}

Let $\mathbf{x}=(\boldsymbol{x}, \psi(\boldsymbol{x})) \in \mathbb{R}^{3}$ denote the earth's surface, where $\boldsymbol{x} \in \mathbb{R}^{2}$ and $\psi: \mathbb{R}^{2} \rightarrow \mathbb{R}$ is a known function for the ground topography. Let $\boldsymbol{\Gamma}(\mathrm{x}, t)$ denote the location of the target at time $t$ located at $\mathbf{x}$ when $t=0$. We assume that $\boldsymbol{\Gamma}(\mathbf{x}, t)$ has the form $\boldsymbol{\Gamma}(\mathbf{x}, t)=\mathbf{x}+\boldsymbol{\Gamma}_{\mathbf{x}}(t)$, where $\boldsymbol{\Gamma}_{\mathbf{x}}(t), \mathbf{x}=[\boldsymbol{x}, \psi(\boldsymbol{x})], \boldsymbol{x} \in$ $\mathbb{R}^{2}$ is a family of one-parameter curves in 3-D space that describes the trajectories of the targets. Thus, for the ground moving targets, $\boldsymbol{\Gamma}_{\mathbf{x} 3}(t)=\psi\left(\left[\boldsymbol{\Gamma}_{\mathbf{x} 1}(t), \boldsymbol{\Gamma}_{\mathbf{x} 2}(t)\right]\right)$, where $\boldsymbol{\Gamma}_{\mathbf{x}}(t)=$ $\left[\boldsymbol{\Gamma}_{\mathbf{x} 1}(t), \boldsymbol{\Gamma}_{\mathbf{x} 2}(t), \boldsymbol{\Gamma}_{\mathbf{x} 3}(t)\right]$. Note that, for stationary targets, $\Gamma_{\mathbf{x}}(t)=0$ for all $t \geq 0$.

Let $\mathbf{v}_{\mathbf{x}}$ denote the velocity of the moving scatterer ${ }^{1}$ located at $\mathbf{x}$ at $t=0$. Then

$$
\mathbf{v}_{\mathbf{x}}=\dot{\boldsymbol{\Gamma}}_{\mathbf{x}}(t)=\left[\boldsymbol{v}_{\mathbf{x}}, \nabla_{\boldsymbol{x}} \psi(\boldsymbol{x}) \cdot \boldsymbol{v}_{\mathbf{x}}\right]
$$

where $\boldsymbol{v}_{\mathbf{x}} \in \mathbb{R}^{2}$ is the 2-D velocity, $\boldsymbol{v}_{\mathbf{x}}=\left[\dot{\boldsymbol{\Gamma}}_{\mathbf{x} 1}(t), \dot{\boldsymbol{\Gamma}}_{\mathbf{x} 2}(t)\right]$, and $\nabla_{\boldsymbol{x}} \psi(\boldsymbol{x})=\left[\begin{array}{ll}\left(\partial \psi / \partial x_{1}\right) & \left(\partial \psi / \partial x_{2}\right)\end{array}\right]$. Note that $\mathbf{v}_{\mathbf{x}}$ may be $t$ dependent. In the rest of our discussion, we make the assumption that the scatterers are moving linearly, and therefore, the velocity $\mathbf{v}_{\mathbf{x}}$ is time independent. We refer to $\mathbf{v}_{\mathbf{x}}$ (or $\boldsymbol{v}_{\mathbf{x}}$ ) for all $\mathrm{x}$ as the velocity field of the scene. We define a model for the velocity field as follows:

$$
\mathbf{v}_{\mathbf{x}}=\sum_{i=1}^{N} \mathbf{v}_{\mathbf{x}_{i}} \varphi\left(\mathbf{x}-\mathbf{x}_{i}\right)
$$

where $\varphi\left(\boldsymbol{x}-\boldsymbol{x}_{i}\right)$ is a smooth differentiable function that approximates Dirac-delta distribution $\delta\left(\mathbf{x}-\mathbf{x}_{i}\right)$ in the limit, and $N$ represents the number of distinct moving targets in the scene. An example for $\varphi\left(\mathbf{x}-\mathbf{x}_{i}\right)$ is $e^{-\left(\left|\mathbf{x}-\mathbf{x}_{i}\right|^{2} / \sigma_{i}\right)}, \sigma_{i} \ll 1$, where $\sigma_{i}$ can be chosen based on the image resolution and the physical extend of the targets. Note that, for well separated targets, as $\mathbf{x} \rightarrow \mathbf{x}_{i}$, so does $\mathbf{v}_{\mathbf{x}} \rightarrow \mathbf{v}_{\mathbf{x}_{i}}$.

We assume that the scattering takes place in a thin region near the ground. Thus, the reflectivity function of the scene at time $t=0$ has the form

$$
V(\mathbf{x})=\rho(\boldsymbol{x}) \delta\left(x_{3}-\psi(\boldsymbol{x})\right) .
$$

At time $t$, the scatter is located at $\mathbf{z}=\mathbf{x}+\mathbf{v}_{\mathbf{x}} t$. The reflectivity function of the moving scatterers translates as

$$
V\left(\mathbf{z}-\mathbf{v}_{\mathbf{x}} t\right) \simeq \rho\left(\boldsymbol{z}-\boldsymbol{v}_{\mathbf{x}} t\right) \delta\left(z_{3}-\psi(\boldsymbol{z})\right) \delta\left(v_{3}-\nabla_{\boldsymbol{z}} \psi(\boldsymbol{z}) \cdot \boldsymbol{v}_{\mathbf{x}}\right)
$$

\footnotetext{
${ }^{1}$ We use the term scatterer for "a point target" and assume that a target is composed of a continuum of scatterers.
} 


\section{B. Received Signal}

Let $\gamma_{T}(t)$ and $\gamma_{R}(t)$ be the transmitter and receiver trajectories, respectively, and let $p(t)$ and $r(t)$ denote the transmitted waveform and the received signal, respectively. We consider a finite and relatively short receiving time-window starting at time $t=s$.

Using the scalar wave equation along with (4) and under the Born approximation, we obtain

$$
\begin{gathered}
r(t+s)=\int \frac{\delta\left(t-t^{\prime}-\left|\gamma_{R}(t+s)-\mathbf{z}\right| / c_{0}\right)}{4 \pi\left|\gamma_{R}(t+s)-\mathbf{z}\right|} V\left(\mathbf{z}-\mathbf{v}_{\mathbf{x}} t\right) \\
\times \frac{\delta\left(t^{\prime}-t^{\prime \prime}-\left|\mathbf{z}-\gamma_{T}\left(t^{\prime \prime}+s\right)\right| / c_{0}\right)}{4 \pi\left|\mathbf{z}-\gamma_{T}\left(t^{\prime \prime}+s\right)\right|} \ddot{p}\left(t^{\prime \prime}+s\right) d t^{\prime \prime} d t^{\prime} d \mathbf{z} .
\end{gathered}
$$

Making the change of variables, i.e., $\mathbf{z} \rightarrow \mathbf{x}$, taking into account the fact that the velocities of the antennas and targets are much less than the speed of light, and under the assumption that the range variation due to the movement of the antennas and the scene is much less than the transmitter-to-scene and scene-to-receiver range, we have [33]

$$
r(t+s)=\omega_{0}^{2} \int \frac{\tilde{p}(\alpha t-\tau+s) e^{\mathrm{i} \omega_{0}(\alpha t-\tau+s)} \rho(\boldsymbol{x})}{(4 \pi)^{2} G_{T R}\left(s, \boldsymbol{x}, \boldsymbol{v}_{\mathbf{x}}\right)} d \boldsymbol{x} .
$$

Note that a narrowband waveform is assumed in (6), i.e., $p(t)=$ $e^{\mathrm{i} \omega_{0} t} \tilde{p}(t)$, where $\omega_{0}$ denotes the carrier frequency, and $\tilde{p}(t)$ is the complex envelope of $p$, which is slow varying as a function of $t$ as compared to $e^{\mathrm{i} \omega_{0} t}$.

In (6), the time dilation $\alpha$ is given by

$$
\begin{aligned}
\alpha=\frac{\left.1-\left[\gamma_{R}(s) \widehat{-(\mathbf{x}}+\mathbf{v}_{\mathbf{x}} s\right)\right] \cdot \dot{\gamma}_{R}(s) / c_{0}}{\left.1+\left[\gamma_{T}(s) \widehat{-(\mathbf{x}}+\mathbf{v}_{\mathbf{x}} s\right)\right] \cdot \dot{\gamma}_{T}(s) / c_{0}} \\
\cdot \frac{\left.1+\left[\gamma_{T}(s) \widehat{-(\mathbf{x}}+\mathbf{v}_{\mathbf{x}} s\right)\right] \cdot \mathbf{v}_{\mathbf{x}} / c_{0}}{\left.1-\left[\gamma_{R}(s) \widehat{-(\mathbf{x}}+\mathbf{v}_{\mathbf{x}} s\right)\right] \cdot \mathbf{v}_{\mathbf{x}} / c_{0}}
\end{aligned}
$$

the time delay $\tau$ is given by

$$
\begin{aligned}
\tau & \approx\left[\left|\gamma_{T}(s)-\left(\mathbf{x}+\mathbf{v}_{\mathbf{x}} s\right)\right|+\left|\gamma_{R}(s)-\left(\mathbf{x}+\mathbf{v}_{\mathbf{x}} s\right)\right|\right] / c_{0} \\
& -\left\{\left[\left(\gamma_{T}(s) \widehat{-\left(\mathbf{x}+\mathbf{v}_{\mathbf{x}} s\right)}\right)+\left(\gamma_{R}(s) \widehat{-\left(\mathbf{x}+\mathbf{v}_{\mathbf{x}} s\right)}\right)\right] \cdot \mathbf{v}_{\mathbf{x}} s\right\} / c_{0}
\end{aligned}
$$

and $G_{T R}$ is the product of the geometrical spreading factors given by

$$
G_{T R}\left(s, \boldsymbol{x}, \boldsymbol{v}_{\mathbf{x}}\right)=\left|\gamma_{R}(s)-\left(\mathbf{x}+\mathbf{v}_{\mathbf{x}} s\right)\right|\left|\mathbf{x}+\mathbf{v}_{\mathbf{x}} s-\gamma_{T}(s)\right| .
$$

Since $\tilde{p}$ is a slow-varying function of time, we approximate $\tilde{p}(\alpha t) \approx \tilde{p}(t)$ in the rest of our discussion.

Note that the time dilation factor $\alpha$ can be approximated as $\alpha=1+\beta$, where $f_{0} \beta\left(f_{0}=\omega_{0} / 2 \pi\right)$ represents the total Doppler frequency induced by the relative radial motion of the antennas and the moving scatters. We refer to it as the bistatic Doppler frequency for moving targets and denote it by $f_{d}\left(s, \boldsymbol{x}, \boldsymbol{v}_{\mathbf{x}}\right)$, i.e.,

$$
\begin{aligned}
f_{d}^{\boldsymbol{v}}\left(s, \boldsymbol{x}, \boldsymbol{v}_{\mathbf{x}}\right) & =\frac{f_{0}}{c_{0}}\left[\left(\gamma_{T}(s) \widehat{-(\mathbf{x}}+\mathbf{v}_{\mathbf{x}} s\right)\right) \cdot\left(\dot{\gamma}_{T}(s)-\mathbf{v}_{\mathbf{x}}\right) \\
& \left.\left.+\left(\gamma_{R}(s) \widehat{-(\mathbf{x}}+\mathbf{v}_{\mathbf{x}} s\right)\right) \cdot\left(\dot{\gamma}_{R}(s)-\mathbf{v}_{\mathbf{x}}\right)\right]
\end{aligned}
$$

In (9) and (10), $\mathbf{x}=[\boldsymbol{x}, \boldsymbol{\psi}(\boldsymbol{x})]$ and $\mathbf{v}_{\mathbf{x}}=\left[\boldsymbol{v}_{\mathbf{x}}, \nabla_{\boldsymbol{x}} \boldsymbol{\psi}(\boldsymbol{x}) \cdot \boldsymbol{v}_{\mathbf{x}}\right]$.

Comparing (6) with the received signal model for conventional wideband SAR, we see that the time dilation term $\alpha$ in (6) is set to 1 in conventional SAR imaging methods of both static or moving scenes. This is due to the fact that conventional wideband SAR image formation methods rely on the "startstop" approximation [25], [26] and the Doppler induced by the movement of antennas and that the targets within each pulse propagation, which is the real "temporal Doppler" as compared with the "spatial Doppler" [34], is ignored. As shown later, our method exploits the temporal Doppler to reconstruct moving target images and to estimate their velocities.

Note that the time dilation term $\alpha$ is composed of two terms: a Doppler scaling term due to the movement of antennas and a Doppler scaling term due to the movement of targets. Similarly, both the time delay $\tau$ and Doppler shift frequency $f_{d}^{\boldsymbol{v}}$ include target velocities. If the scene is stationary, i.e., if $\mathbf{v}_{\mathbf{x}}$ is set to 0 in (7) and (8), the received signal model that we present in (6)-(9) reduces to the one that we introduced in [35] for ultranarrowband CW SAR imaging of stationary scenes. Similarly, the temporal Doppler shift frequency $f_{d}^{\boldsymbol{v}}$ in (10) reduces to the one introduced in [22], whenever $\mathbf{v}_{\mathbf{x}}$ and a certain $\tau^{\prime}$ parameter introduced in [22] are both zero.

\section{Forward Model}

Here, we derive a forward model by correlating the windowed and translated received signal with the scaled or frequency-shifted transmitted waveform. The forward model maps the reflectivity function defined on the 2-D position space to the 2-D correlated data. We use this model to reconstruct reflectivity images of the moving targets and analyze the properties of the reconstructed images.

We define the correlation of the received signal given in (6) with a scaled or frequency-shifted version of the transmitted signal over a finite time window as follows:

$$
d(s, \mu)=\int r(t+s) p^{*}(\mu t) \phi(t) d t
$$

for some $s \in \mathbb{R}$ and $\mu \in \mathbb{R}^{+}$, where $\phi(t), t \in\left[0, T_{\phi}\right]$ is a smooth windowing function with a finite support.

Substituting (6) into (11), we obtain

$d(s, \mu)=\int \frac{e^{\mathrm{i} \omega_{0}(\alpha-\mu) t} e^{\mathrm{i} \omega_{0}(s-\tau)}}{(4 \pi)^{2} G_{T R}\left(s, \boldsymbol{x}, \boldsymbol{v}_{\mathbf{x}}\right)} \omega_{0}^{4} \tilde{p}(t-\tau+s) \tilde{p}^{*}(t) \rho(\boldsymbol{x}) d \boldsymbol{x} d t$.

Note that $\tilde{p}(\mu t) \approx \tilde{p}(t)$ is used in (12). 


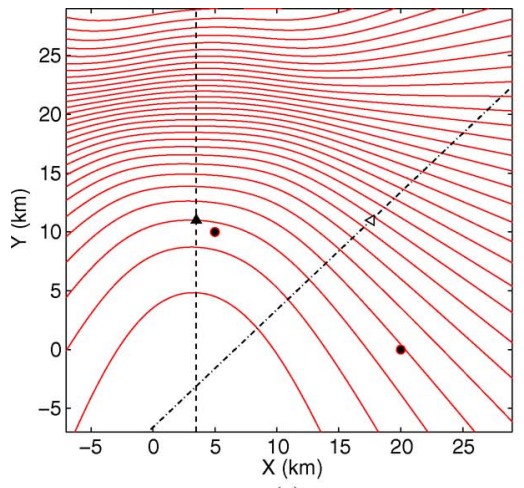

(a)

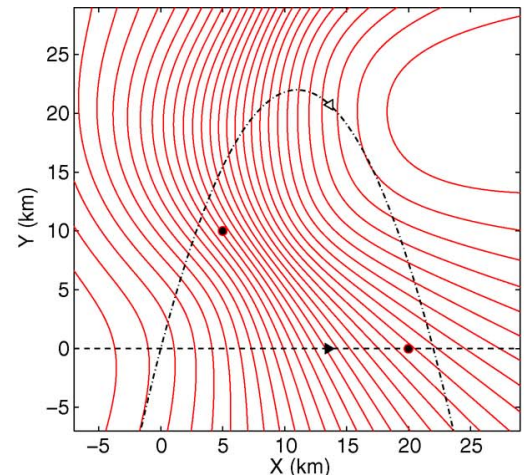

(b)

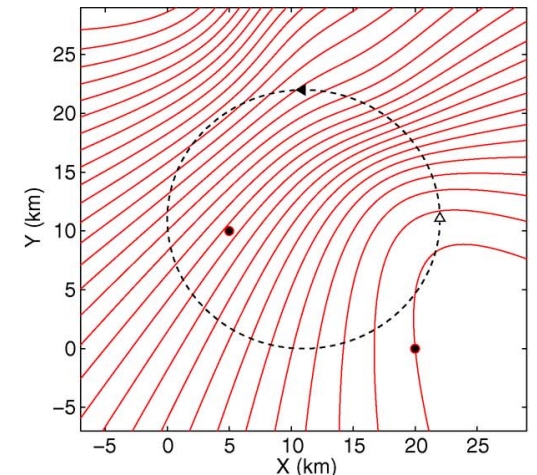

(c)

Fig. 1. Bistatic iso-Doppler contours determined for a certain $s$ and two moving targets located at $\mathbf{x}_{1}=[0.5 e 3,10 e 3,0] \mathrm{m}$ and $\mathbf{x}_{2}=[20 e 3,0,0] \mathrm{m}$, at time $t=0$, moving with velocities $\mathbf{v}_{\mathbf{x}_{1}}=[100,50,0] \mathrm{m} / \mathrm{s}$ and $\mathbf{v}_{\mathbf{x}_{2}}=[0,-200,0] \mathrm{m} / \mathrm{s}$, for three different transmitter and receiver flight trajectories indicated by the dashed and dash-dot lines, respectively. The black and white triangles denote the corresponding positions of the transmitter and receiver for a certain $s$. The black dots indicates the positions of the targets at $t=0$.

We define the forward modeling operator $\mathcal{F}$ from the reflectivity function $\rho$ to correlated data $d$ as follows:

$$
\begin{aligned}
d(s, \mu) & \approx \mathcal{F}_{\boldsymbol{v}}[\rho](s, \mu) \\
& :=\int e^{-\mathrm{i} \phi_{\boldsymbol{v}}(t, \boldsymbol{x}, s, \mu)} A_{\boldsymbol{v}}(t, \boldsymbol{x}, s, \mu) \rho(\boldsymbol{x}) d \boldsymbol{x} d t
\end{aligned}
$$

where

$$
\begin{aligned}
\phi_{\boldsymbol{v}}(t, \boldsymbol{x}, s, \mu) & =2 \pi f_{0} t\left[(\mu-1)+f_{d}^{\boldsymbol{v}}\left(s, \boldsymbol{x}, \boldsymbol{v}_{\mathbf{x}}\right) / f_{0}\right] \\
A_{\boldsymbol{v}}(t, \boldsymbol{x}, s, \mu) & =\frac{\tilde{p}(t-\tau+s) \tilde{p}^{*}(t) e^{\mathrm{i} \omega_{0}(s-\tau)} \omega_{0}^{4}}{(4 \pi)^{2} G_{T R}\left(s, \boldsymbol{x}, \boldsymbol{v}_{\mathbf{x}}\right)}
\end{aligned}
$$

and the bistatic Doppler frequency $f_{d}^{v}$ is defined in (10).

Note that the phase function $\phi_{v}$ and the amplitude term $A_{v}$ in (14) and (15) are not functions of velocity $\boldsymbol{v}_{\mathbf{x}}$. The velocity field $\boldsymbol{v}_{\mathbf{x}}, \boldsymbol{x} \in \operatorname{supp}\left(A_{\boldsymbol{v}}\right)$ is simply fixed and known in $\phi_{\boldsymbol{v}}$ and $A_{v}$.

We assume that $\nabla_{\boldsymbol{x}} \phi_{\boldsymbol{v}} \neq 0$ for all $\boldsymbol{x} \in \operatorname{supp}\left(A_{\boldsymbol{v}}\right)$ and $\partial_{(s, \mu)} \phi_{\boldsymbol{v}} \neq 0$ for all $(s, \mu) \in\left[s_{0}, s_{1}\right] \times \mathbb{R}^{+}$.

Similarly, we assume that, for some $m_{A}, A_{\boldsymbol{v}}$ satisfies the inequality

$$
\begin{aligned}
\sup _{(t, \mu, s, \boldsymbol{x}) \in \mathcal{U}}\left|\partial_{t}^{\alpha_{t}} \partial_{\mu}^{\alpha_{\mu}} \partial_{s}^{\beta_{s}} \partial_{x_{1}}^{\epsilon_{1}} \partial_{x_{2}}^{\epsilon_{2}} A_{\boldsymbol{v}}(t, \boldsymbol{x}, s, \mu)\right| \\
\leq C_{A}\left(1+t^{2}\right)^{\left(m_{A}-\left|\alpha_{t}\right|\right) / 2}
\end{aligned}
$$

where $\mathcal{U}$ is any compact subset of $\mathbb{R} \times \mathbb{R}^{+} \times \mathbb{R} \times \mathbb{R}^{2}$, and the constant $C_{A}$ depends on $\mathcal{U}, \alpha_{t, \mu}, \beta_{s}$, and $\epsilon_{1,2}$. This assumption is needed in order to make various stationary phase calculations hold. Under this assumption, the forward operator $\mathcal{F}_{\boldsymbol{v}}$ can be viewed as a generalized Radon transform [36]. In practice, (16) is satisfied for transmitters and receivers sufficiently far away from the illuminated region.

Note that, when the velocity field is set to zero, the forward model $\mathcal{F}_{\boldsymbol{v}}$ that we define in (13) reduces to the forward model that we introduced in [22] for imaging of stationary scenes using ultranarrowband CW SAR.

\section{Analysis of the Forward Model}

Under the assumptions on the phase, $\phi_{\boldsymbol{v}}$, and (16), the leading-order contribution to the integral in (13) comes from the intersection of the illuminated ground topography and $\mathrm{x} \in \mathbb{R}^{3}$ that have the same bistatic Doppler frequency [36].

We denote the 2-D curves formed by this intersection as

$F_{\boldsymbol{v}}(s, \mu)=\left\{\boldsymbol{x} \in \mathbb{R}^{2}: f_{d}^{\boldsymbol{v}}\left(s, \boldsymbol{x}, \boldsymbol{v}_{\mathbf{x}}\right)=f_{0}(1-\mu), \boldsymbol{x} \in \operatorname{supp}\left(A_{\boldsymbol{v}}\right)\right\}$

and refer to $F_{\boldsymbol{v}}(s, \mu)$ as the bistatic iso-Doppler contours for moving targets.

Fig. 1 shows the bistatic iso-Doppler contours for a scene containing two moving targets and three different flight trajectories over a flat topography: (a) The transmitter and receiver are both traversing straight linear flight trajectories. $\gamma_{T}(t)=[3.5, v t, 6.5] \mathrm{km}$ and $\gamma_{R}(t)=[(v t-7), v t, 6.5] \mathrm{km}$ with speed $v=261 \mathrm{~m} / \mathrm{s}$. (b) The transmitter is traversing a straight linear flight trajectory $\gamma_{R}(t)=[v t, 0,6.5] \mathrm{km}$, and the receiver is traversing a parabolic flight trajectory $\gamma_{T}(t)=$ $\left[v t,(v t-11)^{2} * 22 / 121,6.5\right] \mathrm{km}$ with speed $v=261 \mathrm{~m} / \mathrm{s}$. (c) The transmitter and receiver are both traversing a circular flight trajectory. $\gamma_{T}(\theta)=\gamma_{C}(\theta)$ and $\gamma_{R}(\theta)=\gamma_{C}(\theta-\pi / 2)$, where $\gamma_{C}(\theta)=[11+11 \cos \theta, 11+11 \sin \theta, 6.5] \mathrm{km}$ with $\theta=$ $(v / R) t$, where speed $v=261 \mathrm{~m} / \mathrm{s}$ and radius $R=11 \mathrm{~km}$. We assume that the two moving targets are centered at positions $\mathbf{x}_{1}=[0.5 e 3,10 e 3,0] \mathrm{m}$ and $\mathbf{x}_{2}=[20 e 3,0,0] \mathrm{m}$, at time $t=$ 0 , moving with velocities $\mathbf{v}_{\mathbf{x}_{1}}=[100,50,0] \mathrm{m} / \mathrm{s}$ and $\mathbf{v}_{\mathbf{x}_{2}}=$ $[0,-200,0] \mathrm{m} / \mathrm{s}$, respectively. ${ }^{2}$ We assume that the velocity field $\mathbf{v}_{\mathbf{x}}$ for the scene can be approximated by the following smooth function $\mathbf{v}_{\mathbf{x}_{1}} e^{-\left|\mathbf{x}-\mathbf{x}_{1}\right|^{2} / \sigma^{2}}+\mathbf{v}_{\mathbf{x}_{2}} e^{-\left|\mathbf{x}-\mathbf{x}_{2}\right|^{2} / \sigma^{2}}$, where $\sigma \ll 1$ is a constant. Note that the iso-Doppler contours depend on $s$, as defined in (10). The contours shown in Fig. 1 is for a certain $s$ value. These contours change as the transmitter and receiver and the target(s) move along their trajectories.

\footnotetext{
${ }^{2}$ In order to highlight the impact of the target velocity on the bistatic iso-Doppler contours and show the difference with the bistatic iso-Doppler contours for static scene imaging [22], we used exaggerated velocities.
} 


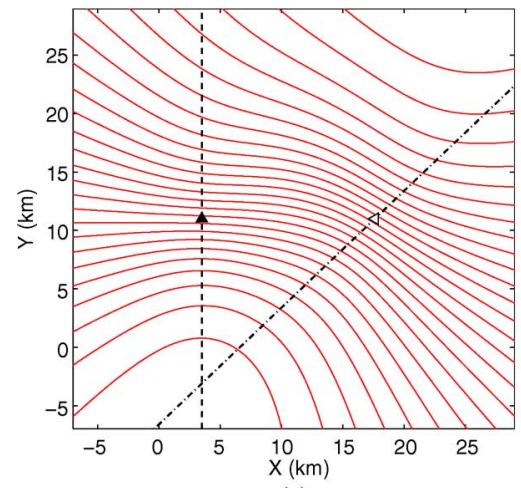

(a)

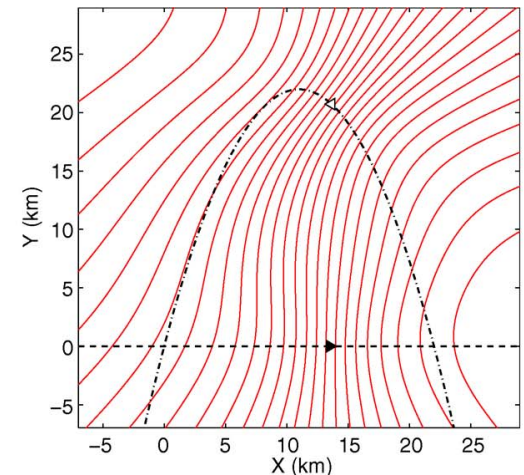

(b)

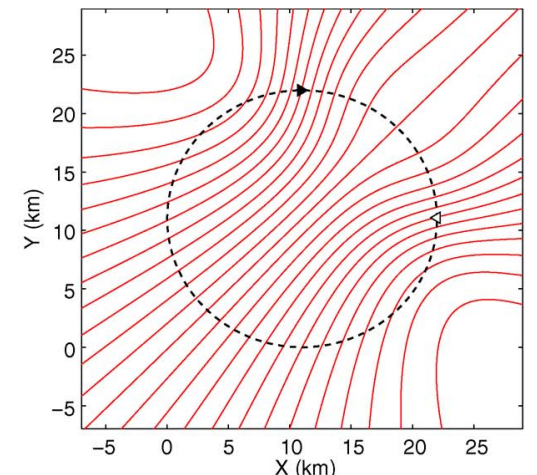

(c)

Fig. 2. Bistatic iso-Doppler contours determined for a certain $s$ when the scene is stationary. The three different flight trajectories of the transmitter and receiver along with the notations are as described in Fig. 1. Note that the visual comparison in Fig. 1 and the figures on top shows that the iso-Doppler contours are warped when the scene includes moving targets.

Fig. 2 shows the iso-Doppler contours when the scene is stationary with the same antenna trajectories as in Fig. 1. Visual comparison of these contours shows that the iso-Doppler contours are warped versions of the contours obtained for a stationary scene when the scene includes moving targets.

The analysis of the forward model $\mathcal{F}_{\boldsymbol{v}}$ shows that the windowed, scaled, and translated correlations between the received signal and the transmitted signal are the projections of the scene reflectivity onto the bistatic iso-Doppler contours for moving targets. We now define imaging of moving targets as the reconstruction of the scene reflectivity $\rho$ and the estimation of the target velocities $\mathbf{v}_{\mathbf{x}}$ based on the forward model $\mathcal{F}_{\boldsymbol{v}}$ using the data $d$.

\section{IMAGE FORMATION}

Here, we first present an FBP-type reflectivity image reconstruction method by backprojecting the data $d$ onto the bistatic iso-Doppler contours for moving targets, under the assumption that the velocity field of the scene is known. We show that the FBP method results in a PSF that can be approximated by a band-limited Dirac-delta function whenever the motion is compensated with the correct velocity field. However, since the velocity field of the scene is unknown, we next backproject the data onto the bistatic iso-Doppler contours for a constant velocity field for a range of velocities. We then show that the position of a target (or targets) whose true velocity is equal to the constant hypothesized velocity lies at the intersection of the bistatic iso-Doppler contours corresponding to the constant velocity field and the bistatic iso-Doppler contours corresponding to the true velocity field of the scene for each $s$ value. Hence, when the data are backprojected onto the constant field iso-Doppler contours, only the targets whose velocity is equal to the hypothesized constant velocity emerge as focused and other targets either do not appear or appear as smeared. We then take an approach similar to [6], [10], [15], and [17] and reconstruct a stack of reflectivity images corresponding to a range of velocities suitable for ground moving targets. We next estimate the velocity of each scatterer from this stack of images using two different figures of merit that measure how well each reflectivity image is focused.

\section{A. Reflectivity Image Reconstruction}

We form reflectivity images of the scene by filtering and backprojecting the correlated data $d$ onto the bistatic isoDoppler contours for moving targets as follows:

$$
\begin{aligned}
\tilde{\rho}(\boldsymbol{z}) & :=\mathcal{K}_{\boldsymbol{v}}[d](\boldsymbol{z}) \\
& =\int e^{\mathrm{i} \phi_{\boldsymbol{v}}(t, \boldsymbol{z}, \mu)} Q_{\boldsymbol{v}}(\boldsymbol{z}, t, s) d(s, \mu) d t d s d \mu
\end{aligned}
$$

where $\mathcal{K}_{\boldsymbol{v}}$ is the FBP operator, and $Q_{v}$ is the filter to be determined below. Note that $\boldsymbol{v}=\boldsymbol{v}_{\mathrm{x}}$ is the fixed and known velocity field in $\phi_{v}$ and $Q_{v}$. Thus, we refer to $\mathcal{K}_{v}$ as the motioncompensated imaging operator in the rest of our discussion.

We assume that, for some $m_{Q_{v}}, Q_{v}$ satisfies the inequality

$$
\begin{aligned}
\sup _{(t, \mu, s, \boldsymbol{z}) \in \mathcal{U}}\left|\partial_{t}^{\alpha_{t}} \partial_{\mu}^{\alpha_{\mu}} \partial_{s}^{\beta_{s}} \partial_{z_{1}}^{\epsilon_{1}} \partial_{z_{2}}^{\epsilon_{2}} Q_{\boldsymbol{v}}(t, \boldsymbol{z}, s, \mu)\right| \\
\leq C_{Q_{\boldsymbol{v}}}\left(1+t^{2}\right)^{\left(m_{Q_{\boldsymbol{v}}}-\left|\alpha_{t}\right|\right) / 2}
\end{aligned}
$$

where $\mathcal{U}$ is any compact subset of $\mathbb{R} \times \mathbb{R}^{+} \times \mathbb{R} \times \mathbb{R}^{2}$, and the constant $C_{Q_{v}}$ depends on $\mathcal{U}, \alpha_{t, \mu}, \beta_{s}$, and $\epsilon_{1,2}$. Under the assumption (19), (18) defines $\mathcal{K}_{\boldsymbol{v}}$ as a Fourier integral operator.

Note that, if the scene is stationary, the backprojection operator defined in (18) reduces to the one introduced in [22], when a certain parameter $\tau^{\prime}$ is also set to 0 .

\section{B. PSF Analysis}

Substituting (13) into (18), we rewrite (18) as

$$
\begin{aligned}
\tilde{\rho}(\boldsymbol{z}) & :=\mathcal{K}_{\boldsymbol{v}} \mathcal{F}_{\boldsymbol{v}}[\rho](\boldsymbol{z}) \\
& =\int L_{\boldsymbol{v}}(\boldsymbol{z}, \boldsymbol{x}) \rho(\boldsymbol{x}) d \boldsymbol{x}
\end{aligned}
$$

where $L_{\boldsymbol{v}}(\boldsymbol{z}, \boldsymbol{x})$ is the PSF of the motion-compensated reflectivity imaging operator given by

$$
\begin{aligned}
L_{\boldsymbol{v}}(\boldsymbol{z}, \boldsymbol{x})=\int e^{\mathrm{i}\left[\phi_{\boldsymbol{v}}(t, \boldsymbol{z}, s, \mu)-\phi_{\boldsymbol{v}}(t, \boldsymbol{x}, s, \mu)\right]} & \\
& \times Q_{\boldsymbol{v}}(\boldsymbol{z}, t, s) A_{\boldsymbol{v}}\left(t^{\prime}, \boldsymbol{x}, s, \mu\right) d t d s d \mu d t^{\prime} .
\end{aligned}
$$




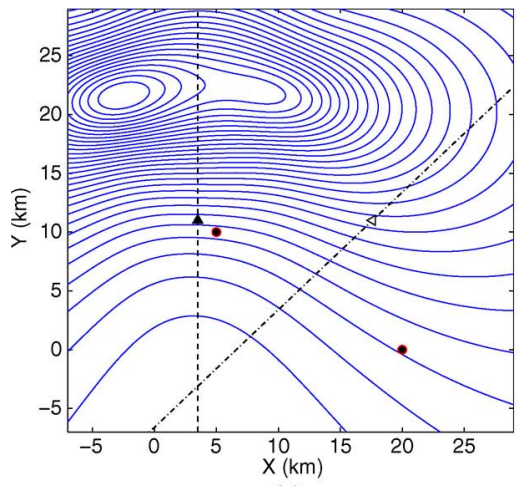

(a)

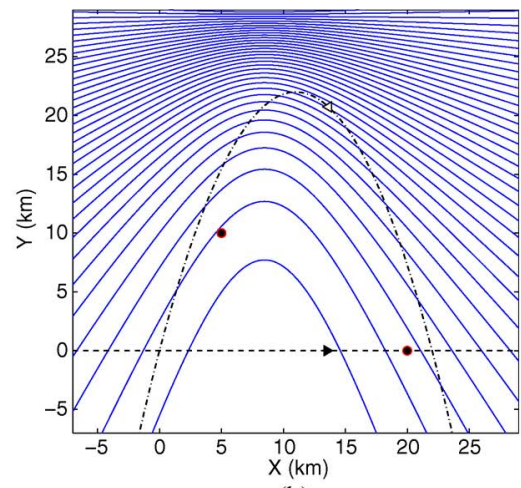

(b)

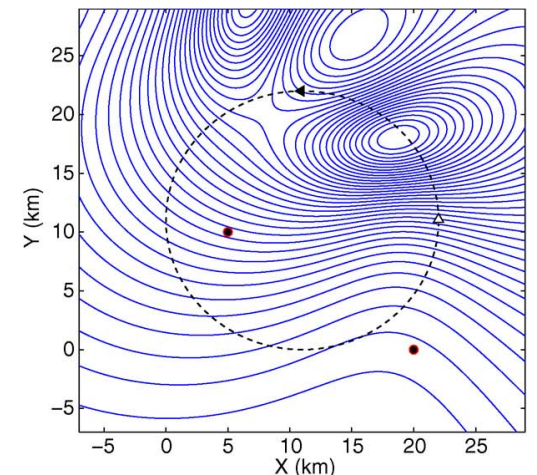

(c)

Fig. 3. Bistatic iso-Doppler-rate contours determined for a certain $s$ and two moving targets, as described in Fig. 1, for three different transmitter and receiver flight trajectories, as described in Section II-D. The black and white triangles denote the corresponding positions of the transmitter and receiver. The black dots indicate the positions of the targets at $t=0$.

We define

$$
\begin{aligned}
\Phi_{\boldsymbol{v}}= & \phi_{\boldsymbol{v}}(t, \boldsymbol{z}, s, \mu)-\phi_{\boldsymbol{v}}(t, \boldsymbol{x}, s, \mu) \\
= & 2 \pi t\left[(\mu-1) f_{0}+f_{d}\left(s, \boldsymbol{z}, \boldsymbol{v}_{\mathbf{z}}\right)\right] \\
& -2 \pi t^{\prime}\left[(\mu-1) f_{0}+f_{d}\left(s, \boldsymbol{x}, \boldsymbol{v}_{\mathbf{x}}\right)\right] .
\end{aligned}
$$

Applying the stationary phase theorem to approximate the $t^{\prime}$ and $\mu$ integrations in (21), ${ }^{3}$ we obtain $t=t^{\prime}$. Substituting $t=t^{\prime}$ into (21), we get the kernel of the image fidelity operator $\mathcal{K}_{\boldsymbol{v}} \mathcal{F}_{\boldsymbol{v}}$ as follows:

$$
\begin{aligned}
& L_{\boldsymbol{v}}(\boldsymbol{z}, \boldsymbol{x}) \approx \int e^{\mathrm{i} 2 \pi t\left[f_{d}\left(s, \boldsymbol{z}, \boldsymbol{v}_{\mathbf{z}}\right)-f_{d}\left(s, \boldsymbol{x}, \boldsymbol{v}_{\mathbf{x}}\right)\right]} \\
& \quad \times Q_{\boldsymbol{v}}(\boldsymbol{z}, t, s) A_{\boldsymbol{v}}\left(t, \boldsymbol{x}, s, 1-f_{d}\left(s, \boldsymbol{x}, \boldsymbol{v}_{\mathbf{x}}\right) / f_{0}\right) d t d s .
\end{aligned}
$$

To simplify our notation, we let

$$
A_{\boldsymbol{v}}(t, \boldsymbol{x}, s)=A_{\boldsymbol{v}}\left(t, \boldsymbol{x}, s, 1-f_{d}\left(s, \boldsymbol{x}, \boldsymbol{v}_{\mathbf{x}}\right) / f_{0}\right) .
$$

The main contributions to $L_{\boldsymbol{v}}$ comes from the critical points of the phase of $\mathcal{K}_{v} \mathcal{F}_{v}$ that satisfy the following conditions [37]:

$$
\begin{aligned}
& f_{d}\left(s, \boldsymbol{x}, \boldsymbol{v}_{\mathbf{x}}\right)=f_{d}\left(s, \boldsymbol{z}, \boldsymbol{v}_{\boldsymbol{z}}\right) \\
& \dot{f}_{d}\left(s, \boldsymbol{x}, \boldsymbol{v}_{\mathbf{x}}\right)=\dot{f}_{d}\left(s, \boldsymbol{z}, \boldsymbol{v}_{\mathbf{z}}\right)
\end{aligned}
$$

where $\dot{f}_{d}\left(s, \boldsymbol{x}, \boldsymbol{v}_{\mathbf{x}}\right)$ denotes the first-order derivative of $f_{d}\left(s, \boldsymbol{x}, \boldsymbol{v}_{\mathbf{x}}\right)$ with respect to $s$. We refer to $\dot{f}_{d}\left(s, \boldsymbol{x}, \boldsymbol{v}_{\mathbf{x}}\right)$ as the bistatic Doppler rate for moving targets.

Using (10), we obtain

$$
\begin{aligned}
\dot{f}_{d}\left(s, \boldsymbol{x}, \boldsymbol{v}_{\mathbf{x}}\right)=\frac{f_{0}}{c_{0}}[ & \frac{1}{\left|\boldsymbol{\gamma}_{T}(s)-\left(\mathbf{x}+\mathbf{v}_{\mathbf{x}} s\right)\right|}\left|\left(\dot{\boldsymbol{\gamma}}_{T}(s)-\mathbf{v}_{\mathbf{x}}\right)_{\perp}\right|^{2} \\
& \left.+\left(\boldsymbol{\gamma}_{T}(s) \widehat{-(\mathbf{x}}+\mathbf{v}_{\mathbf{x}} s\right)\right) \cdot \ddot{\gamma}_{T}(s) \\
& +\frac{1}{\left|\gamma_{R}(s)-\left(\mathbf{x}+\mathbf{v}_{\mathbf{x}} s\right)\right|}\left|\left(\dot{\gamma}_{R}(s)-\mathbf{v}_{\mathbf{x}}\right)_{\perp}\right|^{2} \\
& \left.\left.+\left(\gamma_{R}(s) \widehat{-(\mathbf{x}}+\mathbf{v}_{\mathbf{x}} s\right)\right) \cdot \ddot{\gamma}_{R}(s)\right]
\end{aligned}
$$

\footnotetext{
${ }^{3}$ The determinant of the Hessian of $\Phi_{v}$ is $(2 \pi)^{2} f_{0}^{2}$. Thus, the stationary points are nondegenerate.
}

where

$$
\begin{gathered}
\left.\left(\dot{\gamma}_{T, R}(s)-\mathbf{v}_{\mathbf{x}}\right)_{\perp}=\left(\dot{\gamma}_{T, R}(s)-\mathbf{v}_{\mathbf{x}}\right)-\left(\gamma_{T, R}(s) \widehat{-(\mathbf{x}}+\mathbf{v}_{\mathbf{x}} s\right)\right) \\
\times\left[\left(\gamma_{T, R}(s)-\left(\mathbf{x}+\mathbf{v}_{\mathbf{x}} s\right)\right) \cdot\left(\dot{\gamma}_{T, R}(s)-\mathbf{v}_{\mathbf{x}}\right)\right]
\end{gathered}
$$

denotes the projection of the relative velocity $\dot{\gamma}_{T, R}(s)-\mathbf{v}_{\mathbf{x}}$ onto the plane whose normal vector is along the antenna look direction, i.e., $\left.\gamma_{T, R}(s) \widehat{-(\mathbf{x}}+\mathbf{v}_{\mathbf{x}} s\right)$. Note that, in (27), $\mathbf{x}=$ $[\boldsymbol{x}, \boldsymbol{\psi}(\boldsymbol{x})]$ and $\mathbf{v}_{\mathbf{x}}=\left[\boldsymbol{v}_{\mathbf{x}}, \nabla_{\boldsymbol{x}} \boldsymbol{\psi}(\boldsymbol{x}) \cdot \boldsymbol{v}_{\mathbf{x}}\right]$.

In (27), the summation of the first two terms in the square bracket corresponds to the relative radial acceleration between the transmitter and the target located at $\mathbf{x}+\mathbf{v}_{\mathbf{x}} s$ at time $s$, whereas the summation of the last two terms in the square bracket corresponds to the relative radial acceleration between the receiver and the target located at $\mathbf{x}+\mathbf{v}_{\mathbf{x}} s$ at time $s$.

We refer to the locus of the points formed by the intersection of the illuminated surface, $[\mathbf{x}, \boldsymbol{\psi}(\boldsymbol{x})]$ and the set $\left\{\mathbf{x} \in \mathbb{R}^{3}\right.$ : $\left.\dot{f}_{d}\left(s, \mathbf{x}, \boldsymbol{v}_{\mathbf{x}}\right)=C\right\}$, for some constant $C$, as the bistatic isoDoppler-rate contours for moving targets and denote it by

$$
\dot{F}_{\boldsymbol{v}}(s, C)=\left\{\boldsymbol{x} \in \mathbb{R}^{2}: \dot{f}_{d}\left(s, \boldsymbol{x}, \boldsymbol{v}_{\mathbf{x}}\right)=C, \boldsymbol{x} \in \operatorname{supp}\left(A_{\boldsymbol{v}}\right)\right\} .
$$

Fig. 3 shows the bistatic iso-Doppler-rate contours for two moving targets over a flat topography with three different flight trajectories that are described in Section II-D. Fig. 4 shows the bistatic iso-Doppler-rate contours for the stationary scene over a flat topography with the same flight trajectories as in Fig. 3. Again, we see that the bistatic iso-Doppler-rate contours are warped when the scene includes moving targets. Note that Figs. 3 and 4 are for a certain $s$ value, as in Figs. 1 and 2.

The critical points $z$ of the phase of $\mathcal{K}_{\boldsymbol{v}} \mathcal{F}_{\boldsymbol{v}}$ that contribute to the reflectivity image formation are those points that lie at the intersection of the bistatic iso-Doppler contours $F_{\boldsymbol{v}}(s, \mu)$ and the bistatic iso-Doppler-rate contours $\dot{F}_{\boldsymbol{v}}(s, C)$. For the correct velocity, i.e., $\boldsymbol{v}_{\mathbf{z}}=\boldsymbol{v}_{\mathbf{x}}$, this intersection contributes to the reconstruction of the visible edges ${ }^{4}$ of the scene. ${ }^{5}$ Note that,

\footnotetext{
${ }^{4}$ The term "visible edges" refers to the edges of a scene reflectivity whose information is acquired by the imaging system given the imaging geometry.

${ }^{5} \mathrm{We}$ assume that the flight trajectory and the illumination patterns are chosen such that the intersection has a single element avoiding artifacts such as the right-left type of ambiguities.
} 


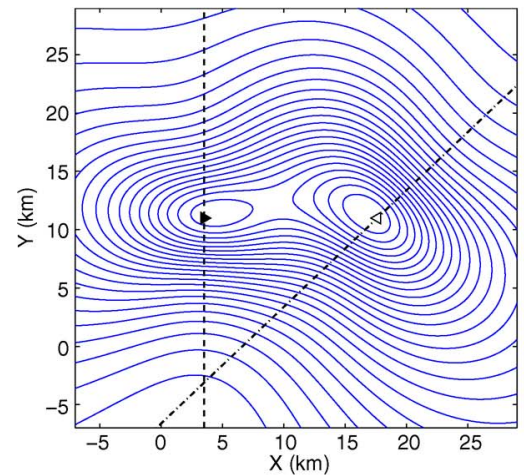

(a)

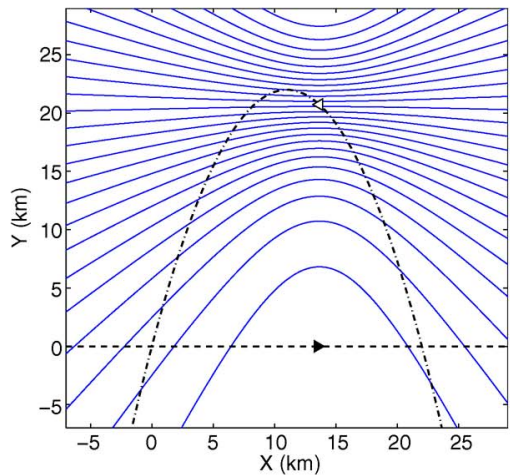

(b)

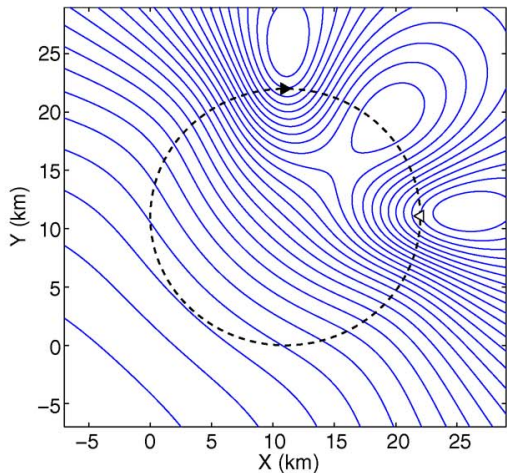

(c)

Fig. 4. Bistatic iso-Doppler-rate contours determined for a certain $s$ when the scene is stationary for three different transmitter and receiver flight trajectories, as described in Section II-D. The black and white triangles denote the corresponding positions of the transmitter and receiver. Note that the iso-Doppler-rate contours are warped version of the figures on top when the scene includes moving targets.

if $\boldsymbol{v}_{\mathbf{z}} \neq \boldsymbol{v}_{\mathrm{x}}$, the points lying at the aforementioned intersection lead to the artifacts in the reconstructed reflectivity image.

\section{Determination of the FBP Filter}

We determine $Q_{v}$ so that the PSF of the motion-compensated reflectivity imaging operator $L_{\boldsymbol{v}}(\boldsymbol{z}, \boldsymbol{x})$ is as close as possible to the Dirac-delta function $\delta(z-x)$ whenever $\boldsymbol{v}_{\mathrm{z}}=\boldsymbol{v}_{\mathrm{x}}$, i.e., whenever the reflectivity at $z$ is reconstructed for the correct target velocity $\boldsymbol{v}_{\mathbf{x}}$. We follow a derivation similar to the one we presented in [22]. We assume that the flight trajectory and the illumination pattern are chosen such that the only contribution to $L_{\boldsymbol{v}}(\boldsymbol{z}, \boldsymbol{x})$ comes from those points $\boldsymbol{z}=\boldsymbol{x}$.

Thus, we linearize $f_{d}\left(s, \boldsymbol{z}, \boldsymbol{v}_{\mathbf{z}}\right)$ around $\boldsymbol{z}=\boldsymbol{x}$ and approximate

$$
f_{d}\left(s, \boldsymbol{z}, \boldsymbol{v}_{\mathbf{z}}\right)-f_{d}\left(s, \boldsymbol{x}, \boldsymbol{v}_{\mathbf{z}}\right) \approx(\boldsymbol{z}-\boldsymbol{x}) \cdot \nabla_{\boldsymbol{z}} f_{d}\left(s, \boldsymbol{z}, \boldsymbol{v}_{\mathbf{z}}\right) .
$$

For each $z$, we make the following change of variables:

$$
(t, s) \rightarrow \boldsymbol{\xi}_{\boldsymbol{v}}=t \nabla_{\boldsymbol{z}} f_{d}\left(s, \boldsymbol{z}, \boldsymbol{v}_{\mathbf{z}}\right)
$$

and write (23) as follows:

$L_{\boldsymbol{v}}(\boldsymbol{z}, \boldsymbol{x})=\int_{\Omega_{\boldsymbol{v}, \boldsymbol{z}}} e^{\mathrm{i}(\boldsymbol{z}-\boldsymbol{x}) \cdot \boldsymbol{\xi}_{\boldsymbol{v}}} Q_{\boldsymbol{v}}\left(\boldsymbol{z}, \boldsymbol{\xi}_{\boldsymbol{v}}\right) A_{\boldsymbol{v}}\left(\boldsymbol{z}, \boldsymbol{\xi}_{\boldsymbol{v}}\right) \eta_{\boldsymbol{v}}\left(\boldsymbol{z}, \boldsymbol{v}_{\mathbf{z}}, \boldsymbol{\xi}_{\boldsymbol{v}}\right) d \boldsymbol{\xi}_{\boldsymbol{v}}$

where

$$
\begin{aligned}
Q_{\boldsymbol{v}}\left(\boldsymbol{z}, \boldsymbol{\xi}_{\boldsymbol{v}}\right) & =Q_{\boldsymbol{v}}\left(\boldsymbol{z}, t\left(\boldsymbol{\xi}_{\boldsymbol{v}}\right), s\left(\boldsymbol{\xi}_{\boldsymbol{v}}\right)\right) \\
A_{\boldsymbol{v}}\left(\boldsymbol{z}, \boldsymbol{\xi}_{\boldsymbol{v}}\right) & =A_{\boldsymbol{v}}\left(t\left(\boldsymbol{\xi}_{\boldsymbol{v}}\right), \boldsymbol{z}, s\left(\boldsymbol{\xi}_{\boldsymbol{v}}\right)\right) \\
\eta_{\boldsymbol{v}}\left(\boldsymbol{z}, \boldsymbol{v}_{\mathbf{z}}, \boldsymbol{\xi}_{\boldsymbol{v}}\right) & =|t|^{-1}\left|\operatorname{det}\left[\begin{array}{c}
\nabla_{\boldsymbol{z}} f_{d}\left(s, \boldsymbol{z}, \boldsymbol{v}_{\mathbf{z}}\right) \\
\partial_{s} \nabla_{\boldsymbol{z}} f_{d}\left(s, \boldsymbol{z}, \boldsymbol{v}_{\mathbf{z}}\right)
\end{array}\right]\right|^{-1}
\end{aligned}
$$

is the determinant of the Jacobian that comes from the change of variables given in (31).

The domain of integration in (32) is given by

$\Omega_{\boldsymbol{v}, \boldsymbol{z}}=\left\{\boldsymbol{\xi}_{\boldsymbol{v}}=t \nabla_{\boldsymbol{z}} f_{d}\left(s, \boldsymbol{z}, \boldsymbol{v}_{\mathbf{z}}\right) \mid A_{\boldsymbol{v}}(t, \boldsymbol{z}, s) \neq 0, \quad t, s \in \mathbb{R}\right\}$
We refer to $\Omega_{\boldsymbol{v}, \boldsymbol{z}}$ as the data collection manifold at $\boldsymbol{z}$ associated with the velocity field $\boldsymbol{v}_{\mathbf{z}}$. This set determines many of the properties of the reconstructed reflectivity image.

We assume that $\left|\nabla_{\mathbf{z}} \boldsymbol{v}_{\mathbf{z}}\right| \approx 0$ almost everywhere in the scene. Using this assumption and (31) and (10), we obtain

$$
\begin{array}{r}
\boldsymbol{\xi}_{\boldsymbol{v}}=-t \frac{2 \pi f_{0}}{c_{0}}\left\{[ D + D ^ { 2 } s ] \cdot \left[\frac{\left(\dot{\gamma}_{T}(s)-\mathbf{v}_{\mathbf{z}}\right)_{\perp}}{\left|\gamma_{T}(s)-\left(\mathbf{z}+\mathbf{v}_{\mathbf{z}} s\right)\right|}\right.\right. \\
\left.+\frac{\left(\dot{\gamma}_{R}(s)-\mathbf{v}_{\mathbf{z}}\right) \perp}{\left|\gamma_{R}(s)-\left(\mathbf{z}+\mathbf{v}_{\mathbf{z}} s\right)\right|}\right] \\
+D^{2} \cdot\left[\left(\gamma_{T}(s) \widehat{\left.-\left(\mathbf{z}+\mathbf{v}_{\mathbf{z}} s\right)\right)}\right.\right. \\
+\left(\gamma_{R}(s) \widehat{\left.\left.-\left(\mathbf{z}+\mathbf{v}_{\mathbf{z}} s\right)\right)\right]}\right\}
\end{array}
$$

where

$$
\begin{aligned}
D & =\left[\begin{array}{lll}
1 & 0 & \partial \psi(\boldsymbol{z}) / \partial z_{1} \\
0 & 1 & \partial \psi(\boldsymbol{z}) / \partial z_{2}
\end{array}\right] \\
D^{2} & =\left[\begin{array}{lll}
0 & 0 & \frac{\partial^{2} \psi(\boldsymbol{z})}{\partial^{2} z_{1}} v_{z 1}+\frac{\partial^{2} \psi(\boldsymbol{z})}{\partial z_{2} \partial z_{1}} v_{z 2} \\
0 & 0 & \frac{\partial^{2} \psi(\boldsymbol{z})}{\partial z_{1} \partial z_{2}} v_{z 1}+\frac{\partial^{2} \psi(\boldsymbol{z})}{\partial^{2} z_{2}} v_{z 2}
\end{array}\right]
\end{aligned}
$$

and $\left(\dot{\gamma}_{T, R}-\mathbf{v}_{\mathbf{z}}\right)_{\perp}$ is the projection of $\dot{\gamma}_{T, R}-\mathbf{v}_{\mathbf{z}}$ onto the plane whose normal is $\left.\gamma_{T, R}(s) \widehat{-(\mathbf{z}}+\mathbf{v}_{\mathbf{z}} s\right)$, as defined by (28). Note that $\mathbf{v}_{\mathbf{z}}=\left[\boldsymbol{v}_{\mathbf{z}}, \nabla_{\boldsymbol{z}} \boldsymbol{\psi}(\boldsymbol{z}) \cdot \boldsymbol{v}_{\mathbf{z}}\right], \boldsymbol{v}_{\mathbf{z}}=\left[v_{z 1}, v_{z 2}\right]$.

For a flat topography, (37) reduces to

$$
\begin{array}{r}
\boldsymbol{\xi}_{\boldsymbol{v}}=-t \frac{2 \pi f_{0}}{c_{0}}\left\{D _ { f } \cdot \left[\frac{\left(\dot{\gamma}_{T}(s)-\mathbf{v}_{\mathbf{z}}\right)_{\perp}}{\left|\gamma_{T}(s)-\left(\mathbf{z}+\mathbf{v}_{\mathbf{z}} s\right)\right|}\right.\right. \\
\left.\left.+\frac{\left(\dot{\gamma}_{R}(s)-\mathbf{v}_{\mathbf{z}}\right)_{\perp}}{\left|\gamma_{R}(s)-\left(\mathbf{z}+\mathbf{v}_{\mathbf{z}} s\right)\right|}\right]\right\}
\end{array}
$$

where $D_{f}=[\mathbf{I}, 0]$ is composed of a $2 \times 2$ identity matrix and a $2 \times 1$ vector of 0 s. For a stationary scene, (40) reduces to

$$
\boldsymbol{\xi}=-t \frac{2 \pi f_{0}}{c_{0}}\left\{D_{f} \cdot\left[\frac{\dot{\gamma}_{T}(s)_{\perp}}{\left|\gamma_{T}(s)-\mathbf{z}\right|}+\frac{\dot{\gamma}_{R}(s)_{\perp}}{\left|\gamma_{R}(s)-\mathbf{z}\right|}\right]\right\}
$$




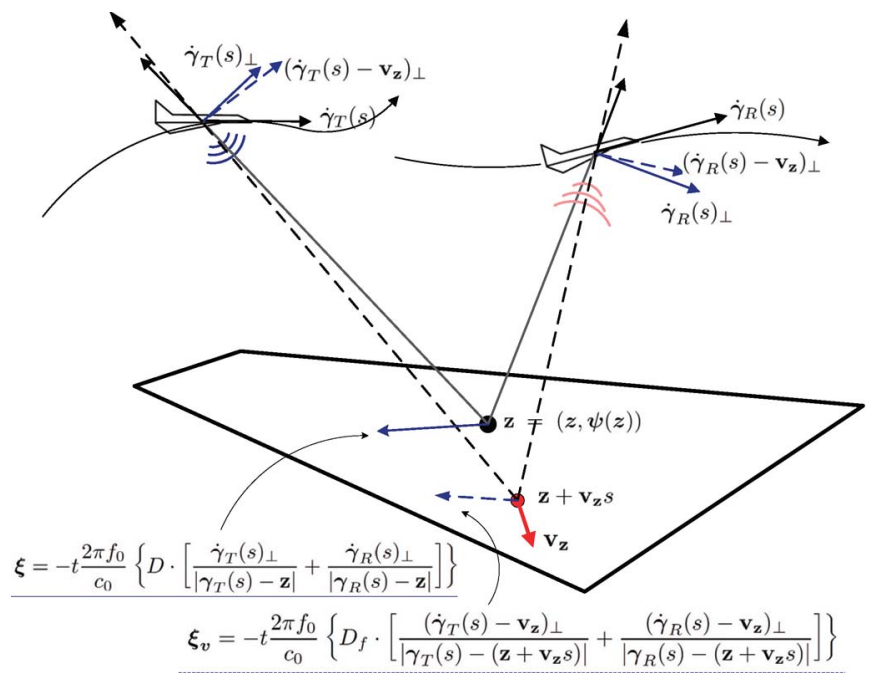

Fig. 5. Illustration of the vectors in (40) and (41), showing the difference between the data collection manifolds in the moving target and the stationary target imaging methods using ultranarrowband CWs. Note that the solid lines represent the vectors involved in the stationary target imaging, whereas the dashed lines represent the vectors involved in the moving target imaging.

where $\dot{\gamma}_{T, R}(s)_{\perp}$ is the projection of $\dot{\gamma}_{T, R}(s)$ onto the plane whose normal is $\gamma_{T, R}(s)-z$. Fig. 5 illustrates the difference between the two vectors given in (40) and (41).

To approximate the PSF $L_{\boldsymbol{v}}(\boldsymbol{z}, \boldsymbol{x})$ in (32) with the Diracdelta function, we choose the filter as follows:

$$
Q_{\boldsymbol{v}}\left(\boldsymbol{z}, \boldsymbol{\xi}_{\boldsymbol{v}}\right)=\frac{\chi_{\Omega_{\boldsymbol{v}, \boldsymbol{z}}}}{\eta_{\boldsymbol{v}}\left(\boldsymbol{z}, \boldsymbol{v}_{\mathbf{z}}, \boldsymbol{\xi}_{\boldsymbol{v}}\right)} \frac{A_{\boldsymbol{v}}^{*}\left(\boldsymbol{z}, \boldsymbol{\xi}_{\boldsymbol{v}}\right)}{\left|A_{\boldsymbol{v}}\left(\boldsymbol{z}, \boldsymbol{\xi}_{\boldsymbol{v}}\right)\right|^{2}}
$$

where $\chi_{\Omega_{v, z}}$ is a smooth cutoff function that prevents division by zero in (42).

With this choice of filter, the resulting motion-compensated FBP operator can recover not only the correct target position and orientation but also the correct strength of the moving target at $\mathbf{z}$.

Note that, for a stationary scene, the filter becomes

$$
Q(\boldsymbol{z}, \boldsymbol{\xi})=\frac{\chi_{\Omega_{z}}}{\eta(\boldsymbol{z}, \boldsymbol{\xi})} \frac{A^{*}(\boldsymbol{z}, \boldsymbol{\xi})}{|A(\boldsymbol{z}, \boldsymbol{\xi})|^{2}}
$$

where $\boldsymbol{\xi}$ is defined as in (40), and $\eta(\boldsymbol{z}, \boldsymbol{\xi})$ is given as

$$
\eta(\boldsymbol{z}, \boldsymbol{\xi})=\left|\frac{\partial(t, s)}{\partial \boldsymbol{\xi}}\right|
$$

and $A(\boldsymbol{z}, \boldsymbol{\xi})=\left.A_{\boldsymbol{v}}(\boldsymbol{z}, \boldsymbol{\xi})\right|_{\boldsymbol{v}_{\mathbf{x}}=0}$, and $\chi_{\Omega_{\boldsymbol{z}}}$ is the indicator function over the support of $A$.

\section{Determination of the Velocity Field}

In developing the FBP operator, we assume that the velocity field of the scene is known a priori. However, in practice, the velocity of a moving target is an unknown quantity and has to be estimated. Below, we describe a method to determine the velocity of moving targets.

We note that the function $\varphi$ and its underlying parameters in the velocity field model $\mathbf{v}_{\mathbf{x}}=\sum_{i=1}^{N} \mathbf{v}_{\mathbf{x}_{i}} \varphi\left(\mathbf{x}-\mathbf{x}_{i}\right)$ are chosen such that, when $\mathbf{x} \rightarrow \mathbf{x}_{i}, \mathbf{v}_{\mathbf{x}} \rightarrow \mathbf{v}_{\mathbf{x}_{i}}$. As a result, the bistatic isoDoppler contours corresponding to a constant field $\mathbf{v}_{\mathbf{x}}=\mathbf{v}_{\mathbf{x}_{i_{0}}}$, i.e., the set of $\boldsymbol{x}$ locations on the ground given by the solution of

$$
f_{d}^{\boldsymbol{v}}\left(s, \boldsymbol{x}, \boldsymbol{v}_{\mathbf{x}_{i_{0}}}\right)=f_{0}(1-\mu)
$$

for all $s$ and $\mu$, include the target location $\boldsymbol{x}_{i_{0}}$, which is also a solution of

$$
f_{d}^{\boldsymbol{v}}\left(s, \boldsymbol{x}, \boldsymbol{v}_{\mathbf{x}}\right)=f_{0}(1-\mu) \quad \text { for } \quad \mathbf{v}_{\mathbf{x}}=\sum_{i=1}^{N} \mathbf{v}_{\mathbf{x}_{i}} \varphi\left(\mathbf{x}-\mathbf{x}_{i}\right)
$$

In other words, the bistatic iso-Doppler contours for the velocity field $\mathbf{v}_{\mathbf{x}}=\mathbf{v}_{\mathbf{x}_{i}}$ and the bistatic iso-Doppler contours corresponding to the velocity field $\mathbf{v}_{\mathbf{x}}=\sum_{i=1}^{N} \mathbf{v}_{\mathbf{x}_{i}} \varphi\left(\mathbf{x}-\mathbf{x}_{i}\right)$ intersect at the target location $\boldsymbol{x}_{i_{0}}$ for all $s$ and $\mu$. Other target locations are either not in the intersection or appear only for some $s$. Therefore, if we backproject the data $d$ onto bistatic isoDoppler contours for a constant hypothesized velocity $\boldsymbol{v}_{h}$, and if this velocity is equal to the velocity of a target (or targets), those targets emerge as focused in the reconstructed image while other targets either do not appear or appear as smeared in the image.

Hence, similar to [6], [10], [15], and [17], we choose a range of hypothesized velocity values $\boldsymbol{v}_{h}$ suitable for ground moving targets. Using the FBP method described in the previous subsections, we reconstruct a reflectivity image corresponding to each velocity $\boldsymbol{v}_{h}$. We refer to this image as the $\boldsymbol{v}_{h^{-}}$ reflectivity image and denote it by $\rho_{\boldsymbol{v}_{h}}(\boldsymbol{z})$. Note that, whenever the hypothesized velocity $\boldsymbol{v}_{h}$ is equal to the true target velocity $\boldsymbol{v}_{\mathrm{x}}$, the backprojection and the filter described in the previous subsections ensure that the visible edge at $\mathbf{x}$ in the scene is reconstructed at the correct location, orientation, and strength. We refer to the image at $\mathbf{z}$ as focused if $\boldsymbol{v}_{h}=\boldsymbol{v}_{\mathbf{x}}$. If, on the other hand, $\boldsymbol{v}_{h} \neq \boldsymbol{v}_{\mathbf{x}}$, the reconstructed edges of the scene are expected to be spread.

In order to determine the velocity of a scatter at $\mathbf{z}$, we measure the degree to which the image $\rho_{\boldsymbol{v}_{h}}(\boldsymbol{z})$ is focused at $\mathbf{z}$ using two different figures of merit. Both of these figures of merit can be viewed as metrics that quantify the spread of edges.

The first figure of merit is the image contrast measure, which can be viewed as the ratio of the standard deviation to the mean of the image [6], [38]. Using this figure of merit, we generate a contrast image as follows:

$$
I\left(\boldsymbol{v}_{h}\right)=\frac{\mathrm{E}\left[\left|\rho_{\boldsymbol{v}_{h}}^{s}-\mathrm{E}\left[\rho_{\boldsymbol{v}_{h}}^{s}\right]\right|^{2}\right]}{\left|\mathrm{E}\left[\rho_{\boldsymbol{v}_{h}}^{s}\right]\right|^{2}}
$$

where $\boldsymbol{v}_{h}=\left[v_{h 1}, v_{h 2}\right]$ is the index of the contrast image, $\rho_{\boldsymbol{v}_{h}}^{s}$ denotes a small neighborhood around the peak of $\rho_{\boldsymbol{v}_{h}}$, and $\mathrm{E}\left[\rho_{\boldsymbol{v}_{h}}^{s}\right]$ denotes the mean value of $\rho_{\boldsymbol{v}_{h}}^{s}$ over its spatial coordinates, i.e., over all pixel values in this neighborhood. Note that this figure of merit was previously used in [6] and [38] to determine target velocities from a stack of images for the conventional SAR moving target imaging.

The second figure of merit is based on the image gradient. Since our reconstruction method is based on recovering the visible edges of the scene, defocusing in the reconstructed 
TABLE I

PARAMETERs That AFFECT THE RESOLUTION OF THE $\boldsymbol{v}_{h}$-REFLECTIVITY IMAGE

\begin{tabular}{lll}
\hline \hline Parameter & Increase $(\uparrow)$ & Resolution \\
\hline Carrier frequency: $f_{0}$ & $\uparrow$ & $\uparrow$ \\
Length of the windows $L_{\phi}$ & $\uparrow$ & $\uparrow$ \\
Distance $\left|\gamma_{T}(s)-\left(\mathbf{z}+\mathbf{v}_{\mathbf{z}} s\right)\right|,\left|\gamma_{T}(s)-\left(\mathbf{z}+\mathbf{v}_{\mathbf{z}} s\right)\right|$ & $\uparrow$ & $\downarrow$ \\
Relative velocity $\dot{\gamma}_{T}-\mathbf{v}_{\mathbf{z}}$ or $\dot{\gamma}_{R}-\mathbf{v}_{\mathbf{z}}$ & $\uparrow$ & $\uparrow$ \\
Bistatic angle $\theta_{T R}$ & $\uparrow$ & $\downarrow$ \\
Ground topography variations & $\uparrow$ & $\uparrow$ \\
Number of $s$ samples & $\uparrow$ & $\uparrow$ \\
\hline \hline
\end{tabular}

Higher $(\uparrow)$ or Lower $(\downarrow)$

images occurs due to the spread of edges. Therefore, a gradientbased metric is a suitable metric to quantify the degree of defocus in reconstructed images.

We define a gradient image as follows:

$$
G\left(\boldsymbol{v}_{h}\right)=\int_{\mathbf{S}_{f}}\left|\nabla_{\boldsymbol{z}} \rho_{\boldsymbol{v}_{h}}(\boldsymbol{z})\right|^{2} d \boldsymbol{z}
$$

where $\mathbf{S}_{f}$ denotes a small region around the peak of $\rho_{\boldsymbol{v}_{h}}$.

Note that we do not necessarily assume that the number of targets $N$ in the scene is known a priori. If there are multiple moving targets with different velocities in the scene, the contrast and gradient images could have several peaks, each one corresponding to the velocity of a different moving target. We accordingly detect the moving targets and determine their velocities by detecting the local maxima in the contrast and gradient images. In practical implementation, since gradient and contrast images are discrete, a threshold-based detection can be used in the absence of prior knowledge about the number of moving targets. The threshold determines the number of the moving targets, whose velocities are estimated by locating the peaks above the threshold in the contrast or gradient image. The threshold can be determined based on the constant-falsealarm-rate criterion [39], which takes into account the statistics of the background clutter.

In practice, the discretized and estimated velocity may deviate from the true velocity. In Section V, we numerically analyze the sensitivity of the image contrast and image gradient measures with respect to small errors in the estimated velocity and with respect to signal-to-clutter-and-noise ratio (SCNR).

\section{Resolution AnAlysis}

Here, we analyze the resolution of reconstructed reflectivity images. Our resolution analysis results are consistent with the Doppler ambiguity theory of ultranarrowband CW signals [40].

To determine the resolution of the reconstructed reflectivity images, we analyze the bandwidth of the PSF associated with the image fidelity operator $\mathcal{K}_{v} \mathcal{F}_{v}$.

Substituting (42) into (32) and the result back into (20), we obtain

$$
\begin{aligned}
\tilde{\rho}(\boldsymbol{z}) & :=\mathcal{K}_{\boldsymbol{v}} \mathcal{F}_{\boldsymbol{v}}[\rho](\boldsymbol{z}) \\
& =\int_{\Omega_{\boldsymbol{v}, \boldsymbol{z}}} e^{\mathrm{i}(\boldsymbol{z}-\boldsymbol{x}) \cdot \boldsymbol{\xi}_{v}} \rho(\boldsymbol{x}) d \boldsymbol{x} d \boldsymbol{\xi}_{\boldsymbol{v}} .
\end{aligned}
$$

Equation (49) shows that the image $\widetilde{\rho}(\boldsymbol{z})$ is a band-limited version of $\rho$, whose bandwidth is determined by the data collection manifold $\Omega_{v, z}$. The larger the data collection manifold, the better the resolution of the reconstructed reflectivity image becomes. Furthermore, as indicated by (36) and (37), the bandwidth contribution of $\boldsymbol{\xi}_{v}$ to the reflectivity image at $\boldsymbol{z}$ is given by

$$
\begin{array}{r}
\frac{2 \pi f_{0}}{c_{0}} L_{\phi} \mid\left[D+D^{2} s\right] \cdot\left[\frac{\left(\dot{\gamma}_{T}(s)-\mathbf{v}_{\mathbf{z}}\right)_{\perp}}{\left|\gamma_{T}(s)-\left(\mathbf{z}+\mathbf{v}_{\mathbf{z}} s\right)\right|}\right. \\
\left.+\frac{\left(\dot{\gamma}_{R}(s)-\mathbf{v}_{\mathbf{z}}\right)_{\perp}}{\left|\gamma_{R}(s)-\left(\mathbf{z}+\mathbf{v}_{\mathbf{z}} s\right)\right|}\right] \\
+2 \cos \frac{\theta_{T R}\left(\mathbf{z}, \mathbf{v}_{\mathbf{z}}, s\right)}{2} D^{2} \cdot \hat{b}_{T R}\left(\mathbf{z}, \mathbf{v}_{\mathbf{z}}, s\right) \mid
\end{array}
$$

where $L_{\phi}$ denotes the length of the support of $\phi(t)$, $\hat{b}_{T R}\left(\mathbf{z}, \mathbf{v}_{\mathbf{z}}, s\right)$ denotes the unit vector in the direction of

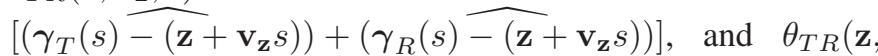
$\left.\mathbf{v}_{\mathbf{z}}, s\right)$ denotes the bistatic angle formed by the transmitter and receiver with respect to the target located at $\left(\mathbf{z}+\mathbf{v}_{\mathbf{z}} s\right)$ at time s. $D$ and $D^{2}$ are as described in (38) and (39).

Equation (50) shows that, as the carrier frequency of the transmitted signal $f_{0}$ becomes higher, the magnitude of $\boldsymbol{\xi}_{v}$ gets larger, which results in higher resolution reflectivity images of the moving target. Furthermore, (50) shows that the resolution depends on the range of the antenna to the moving target via the terms $\left|\gamma_{T}(s)-\left(\mathbf{z}+\mathbf{v}_{\mathbf{z}} s\right)\right|$ and $\left|\gamma_{R}(s)-\left(\mathbf{z}+\mathbf{v}_{\mathbf{z}} s\right)\right|$ and on the relative speed between the transmitter (receiver) and the moving target via the terms $\left(\dot{\gamma}_{T}(s)-\mathbf{v}_{\mathbf{z}}\right)_{\perp}$ and $\left(\dot{\gamma}_{R}(s)-\right.$ $\left.\mathbf{v}_{\mathbf{z}}\right)_{\perp}$. As the antennas move away from the target, or the relative speed decreases in certain directions, the magnitude of $\xi$ decreases, which results in reduced resolution. Additionally, larger number of processing windows, i.e., $s$ samples, used for imaging leads to a larger data collection manifold and, hence, better resolution. As indicated by the second term of (50), the resolution of the reflectivity image also depends on the bistatic angle $\theta_{T R}\left(\mathbf{z}, \mathbf{v}_{\mathbf{z}}, s\right)$. The larger the $\theta_{T R}\left(\mathbf{z}, \mathbf{v}_{\mathbf{z}}, s\right)$, the lower the resolution becomes.

We summarize the parameters that affect the resolution of the reconstructed moving target image in Table I.

\section{Numerical Simulations}

We performed three sets of numerical simulations to demonstrate the performance of our imaging method and to validate 


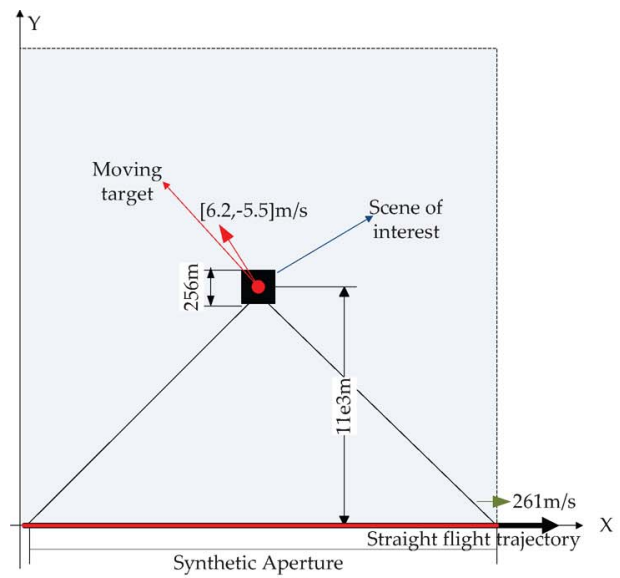

(a)

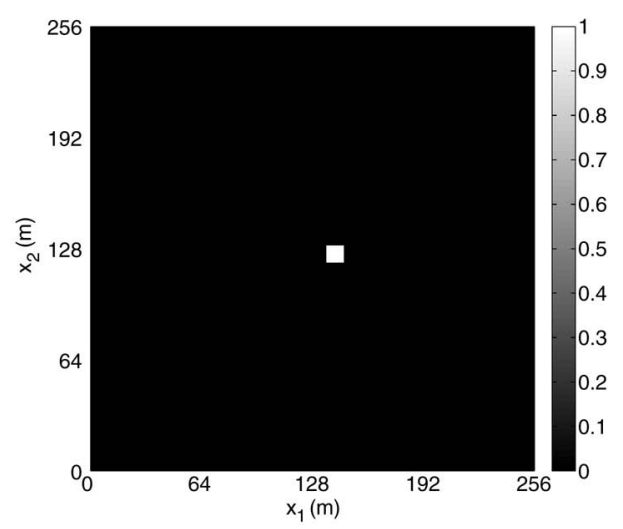

(b)

Fig. 6. (a) Two-dimensional illustration of the simulation setup for a single moving target using a monostatic antenna. The dark region shows the scene

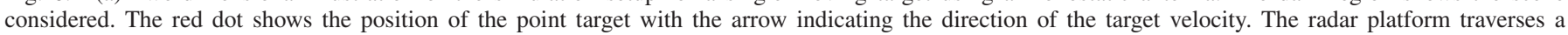
straight linear trajectory, as shown by the black line. The aperture used for the image is shown by the red line. (b) Scene to be reconstructed.

the theoretical results. In the first set of simulations, we numerically studied the reflectivity (or position) reconstruction performance and the velocity estimation performance of our method for a single moving target. We analyzed the sensitivity of the contrast and gradient images with respect to small errors in the estimated velocity due to discretization. We also analyzed the robustness of contrast and gradient metrics in velocity estimation with respect to clutter and noise at different SCNRs. In the second set of simulations, we numerically studied the PSF of the motion-compensated imaging operator developed in Section III under different scenarios and demonstrated the theoretical results of the resolution analysis described in Section IV for flat topography. In the third set of simulations, we demonstrated the performance of our imaging method for multiple moving targets embedded in uniform clutter and structured clutter.

Different transmitter and receiver trajectories were used in the three sets of simulations. In the first two sets of simulations, we considered a monostatic antenna traversing a straight linear trajectory. In the third set of simulations, we considered a bistatic setup where both the transmitter and receiver are traversing the same circular trajectory in tandem with an initial position offset.

For all the numerical experiments, we assumed that a singlefrequency $\mathrm{CW}$ is being transmitted. We used (6) to generate the received signal and (11) to generate the data used for imaging and chose the windowing function $\phi$ in (11) to be a Hanning function.

\section{A. Simulations for a Single Moving Target}

1) Settings: We considered a scene of size $256 \times 256 \mathrm{~m}^{2}$ with flat topography centered at $[11,11,0] \mathrm{km}$. The scene was discretized into $128 \times 128$ pixels, where $[0,0,0] \mathrm{m}$ and $[256,256,0] \mathrm{m}$ correspond to the pixels $(1,1)$ and $(128,128)$, respectively. We assumed that a single square moving target of size $10 \mathrm{~m} \times 10 \mathrm{~m}$ having unit reflectivity was centered at $[142$, $126] \mathrm{m}$, at time $t=0$, corresponding to the $(71,63)$ th pixel in the scene.
We considered a monostatic antenna traversing a straight linear trajectory $\gamma_{L}(v t)=(v t, 0,6.5) \mathrm{km}$ at a constant speed, where $v=261 \mathrm{~m} / \mathrm{s}$ is the radar velocity. Fig. 6(a) shows the 2-D schematic of the scene with the target and antenna trajectories. The aperture length used for the image was $22 \mathrm{e} 3 \mathrm{~m}$, as indicated by the red line. Fig. 6(b) shows the real scene with a moving target at time $t=0$.

We reconstructed $\tilde{\rho}_{\boldsymbol{v}_{h}}(\boldsymbol{z})$ images via the FBP method, as described in Section III and III-D, with $f_{0}=800 \mathrm{MHz}$, $L_{\phi}=0.0107 \mathrm{~s}$, and the aperture sampling frequency $f_{s}=$ $24.2967 \mathrm{~Hz}$.

2) Robustness of Contrast- and Gradient-Based Velocity Estimation Due to Discretization Errors in Velocity: We assumed the moving velocity of the target to be $[6.2,-5.5,0] \mathrm{m} / \mathrm{s}$, as shown in Fig. 6(a). We assumed that the velocity of the target is in the range of $[-10,10] \times[-10,10] \mathrm{m} / \mathrm{s}$ and implemented the velocity estimation in two stages, each one using a different discretization step: We first discretized the entire velocity space into a $21 \times 21$ grid with a step size of $1 \mathrm{~m} / \mathrm{s}$, from which we obtained an initial estimate of the target velocity $\tilde{\boldsymbol{v}}_{\mathbf{x}, 0}$. Then, we discretized a small region of size $[-1,1] \times[-1,1] \mathrm{m} / \mathrm{s}$ around the initial velocity estimate into a $21 \times 21$ grid with a step size of $0.05 \mathrm{~m} / \mathrm{s}$ to refine our velocity estimate obtained at the first stage. Note that, in order to isolate the sensitivity of contrastand gradient-based velocity estimation with respect to errors in velocity discretization, we conducted the simulations here without clutter and noise.

We formed the contrast and gradient images for the first velocity estimation stage, as described in Section III-D. The results are shown in Fig. 7(a) and (b). The black circle indicates the estimated velocity. The initial velocity estimates obtained from Fig. 7(a) and (b) are $\tilde{\boldsymbol{v}}_{\mathbf{x}, 0}^{c}=[7,-5] \mathrm{m} / \mathrm{s}$ and $\tilde{\boldsymbol{v}}_{\mathbf{x}, 0}^{g}=[6,-5] \mathrm{m} / \mathrm{s}$, respectively, both of which are close to the true target velocity $\boldsymbol{v}_{\mathbf{x}}=[6.2,-5.5] \mathrm{m} / \mathrm{s}$. The bright regions around the peaks in the contrast and gradient images shown in Fig. 7(a) and (b) indicate that the image contrast and gradient vary smoothly with the hypothesized velocity.

Comparing the two initial velocity estimates, we find that the image gradient outperforms the image contrast in this 


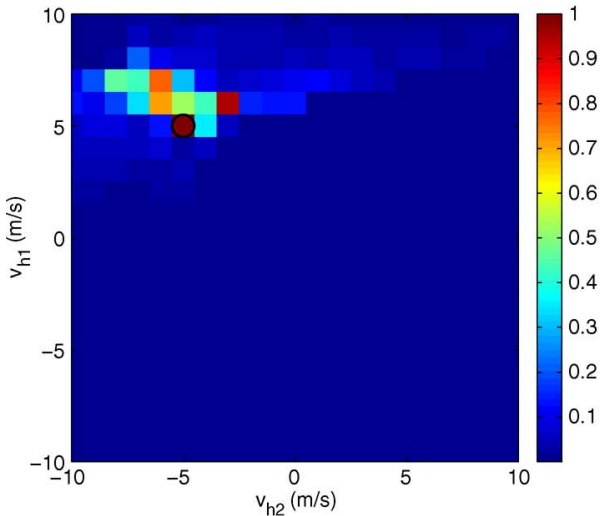

(a)

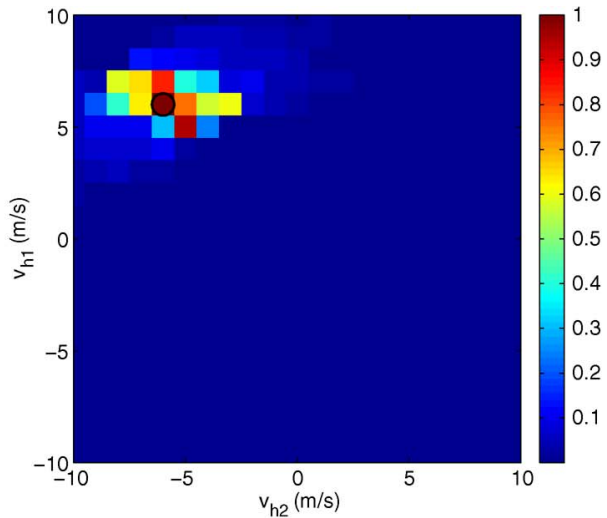

(b)

Fig. 7. (a) Contrast and (b) gradient images formed in the first velocity estimation stage. The two images are formed for the entire velocity space discretized using a step size of $1 \mathrm{~m} / \mathrm{s}$. The estimated velocities are $\tilde{\boldsymbol{v}}_{\mathbf{x}, 0}^{c}=[7,-6] \mathrm{m} / \mathrm{s}$ and $\tilde{\boldsymbol{v}}_{\mathbf{x}, 0}^{g}=[6,-6] \mathrm{m} / \mathrm{s}$, as indicated by the black circles.

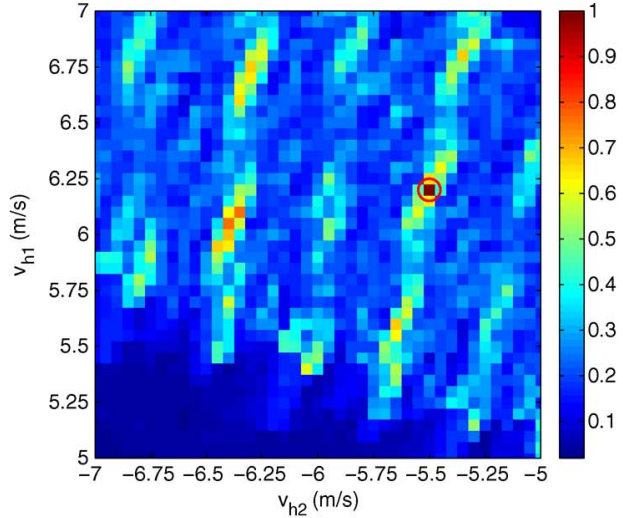

(a)

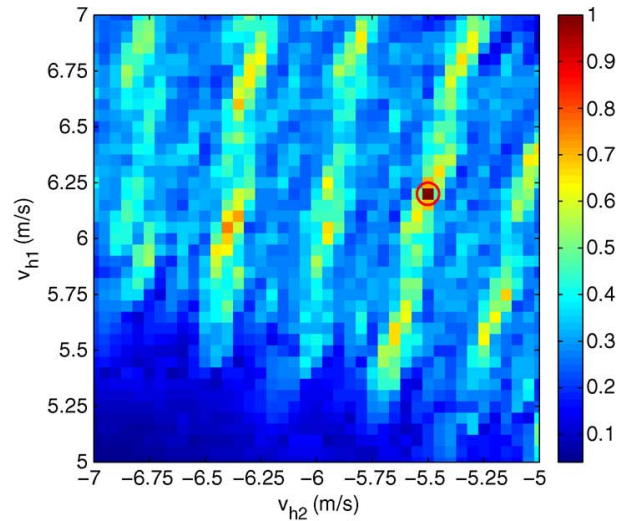

(b)

Fig. 8. (a) Contrast and (b) gradient images formed in the second velocity estimation stage. The two images are formed for a small velocity region of size $[-1,1] \times[-1,1] \mathrm{m} / \mathrm{s}$, around $\tilde{\boldsymbol{v}}_{\mathbf{x}, 0}^{g}=[6,-6] \mathrm{m} / \mathrm{s}$, using a step size of $0.05 \mathrm{~m} / \mathrm{s}$. This corresponds to the region $[5,7] \times[-7,-5] \mathrm{m} / \mathrm{s}$. The estimated velocities in this stage are $\tilde{\boldsymbol{v}}_{\mathbf{x}, 0}^{c}=\tilde{\boldsymbol{v}}_{\mathbf{x}, 0}^{g}=[6.2,-5.5] \mathrm{m} / \mathrm{s}$, as indicated by the red circles.

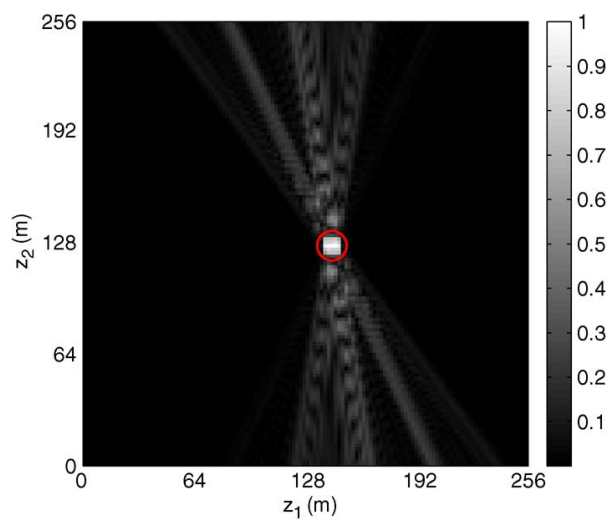

Fig. 9. Reconstructed reflectivity images with the estimated velocity $\tilde{\boldsymbol{v}}_{\mathbf{x}}=$ $[6.2,-5.5] \mathrm{m} / \mathrm{s}$. The red circle indicates the true target position at time $t=0$.

experiment, since $\tilde{\boldsymbol{v}}_{\mathbf{x}, 0}^{g}$ is closer to the true target value. At the second stage of the velocity estimation, we used a finer discretization step around $\tilde{\boldsymbol{v}}_{\mathrm{x}, 0}^{g}$. The resulting contrast and gradient images are shown in Fig. 8(a) and (b), respectively. It is shown that both Fig. 8(a) and (b) result in a velocity estimate of $[6.2,-5.5] \mathrm{m} / \mathrm{s}$ shown by the red circle, which is consistent with the true target velocity. Note that we use the black circle to

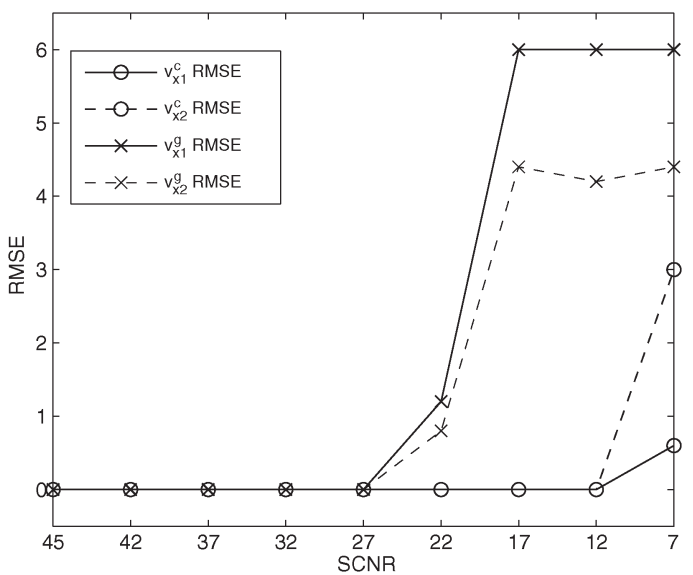

Fig. 10. RMSE of the estimated velocities as a function of SCNR (unit: $\mathrm{dB}$ ) using contrast- and gradient-based velocity estimation, which were indicated by circle and cross markers, respectively. Note that the solid and dashed lines represent the RMSE of $\tilde{\boldsymbol{v}}_{\mathbf{x} 1}$ and $\tilde{\boldsymbol{v}}_{\mathbf{x} 2}$, respectively.

denote the estimated value and the red circle to denote the true value. If the estimated value is almost equal to the true value, only the marker for true value is shown. 


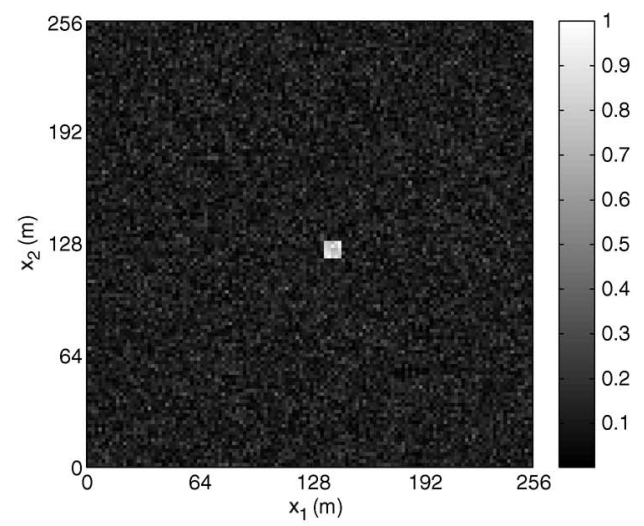

(a)

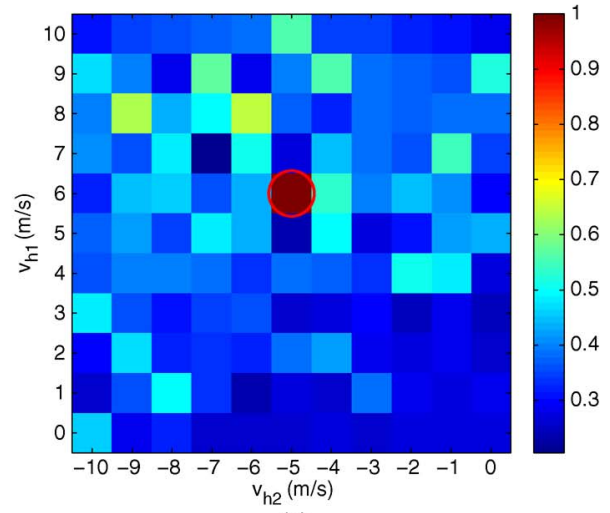

(c)

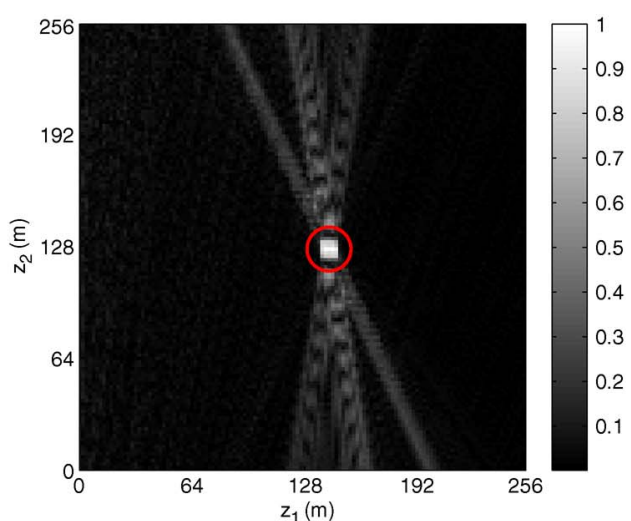

(b)

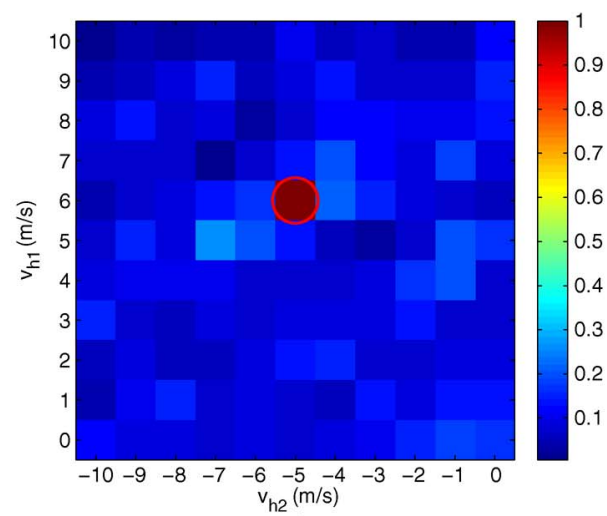

(d)

Fig. 11. Simulation results at high SCNR (32 dB). (a) Original scene. (b) Moving target reflectivity image reconstructed with the estimated velocity $\tilde{\boldsymbol{v}}_{\mathbf{x}}=$ $[6,-5] \mathrm{m} / \mathrm{s}$. The red circle indicates the true target position at time $t=0$. (c) Contrast image. (d) Gradient image. The estimated velocities $\tilde{\boldsymbol{v}}_{\mathbf{x}}^{c}$ and $\tilde{\boldsymbol{v}}_{\mathbf{x}}^{g}$ are both consistent with the true target velocity $\boldsymbol{v}_{\mathbf{x}}$, i.e., $\tilde{\boldsymbol{v}}_{\mathbf{x}}^{c}=\tilde{\boldsymbol{v}}_{\mathbf{x}}^{g}=\boldsymbol{v}_{\mathbf{x}}$, which were indicated by the red circles in (c) and (d).

Fig. 9 shows the reconstructed reflectivity image of the moving target when $\boldsymbol{v}_{h}=\tilde{\boldsymbol{v}}_{\mathbf{x}}=[6.2,-5.5] \mathrm{m} / \mathrm{s}$. We see that the amplitude, position, and orientation of the edges of the moving target are well reconstructed. The red circle shows the true target location.

3) Robustness of Velocity Estimation With Respect to Noise and Clutter: Here, we present the reconstruction and velocity estimation of a moving target using data with clutter and noise at different SCNR levels to analyze the robustness of contrastor gradient-based velocity estimation with respect to clutter and noise. The target velocity was set to $[6,-5,0] \mathrm{m} / \mathrm{s}$, and the range of the hypothesized velocities was assumed to be $[0,10] \times[-10,0] \mathrm{m} / \mathrm{s}$. The entire velocity space was discretized into an $11 \times 11$ grid with a step size of $1 \mathrm{~m} / \mathrm{s}$.

We considered a uniform clutter background [17], [41] and simulated it as an uncorrelated complex Gaussian random field. The receiver noise was simulated as an additive white Gaussian process with zero mean. The clutter-to-noise ratio (CNR) was set to $20 \mathrm{~dB}$. Ten different realizations of noise and clutter were generated to calculate the root-mean-square error (RMSE).

We formed the contrast and gradient images under different SCNRs and estimated the velocity, as described in Section III-D. Fig. 10 shows the RMSE of the estimated velocities using these two figures of merit. We see that the contrast-based metric outperforms the gradient-based metric under low SCNRs, since gradient increases the effect of noise or clutter in images. The contrast-based approach performs well when SCNR $\geq 10 \mathrm{~dB}$, whereas the gradient-based velocity estimation requires higher SCNR ( $25 \mathrm{~dB})$ to achieve the same RMSE performance as the contrast-based approach. Note that the graceful degradation of the RMSE curve as the SCNR decreases indicates that the contrast- and gradient-based metrics are robust with respect to clutter and noise.

Figs. 11 and 12 show the original scenes, the reconstructed moving target images, and the contrast and gradient images for $\mathrm{SCNR}=32 \mathrm{~dB}$ and SCNR $=12 \mathrm{~dB}$, respectively. Fig. 11(c) and (d) shows that under high SCNR, both contrast- and gradient-based metrics lead to correct velocity estimation. Note that, at high SCNR, the gradient image provides a better test statistic than the contrast image for velocity estimation, as can be seen from the clear peak at the correct velocity. However, at low SCNRs, the contrast-based metric is more robust and provides a better test statistic than the gradient-based approach, as shown in Fig. 12(c) and (d). Fig. 12(b) shows the image reconstructed using the contrast-based velocity estimation.

\section{B. Numerical Analysis of the PSF}

Here, we present a numerical analysis of the motioncompensated PSF, i.e., PSF when $\boldsymbol{v}_{h}=\boldsymbol{v}_{\mathbf{x}}$, to verify the theoretical results obtained in Section IV. We used the same simulation geometry and scene setup, as described in Section V-A. We placed a point moving target with unit 


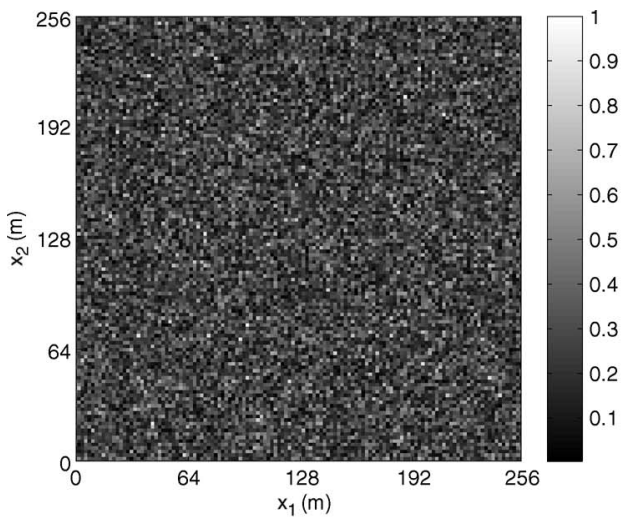

(a)

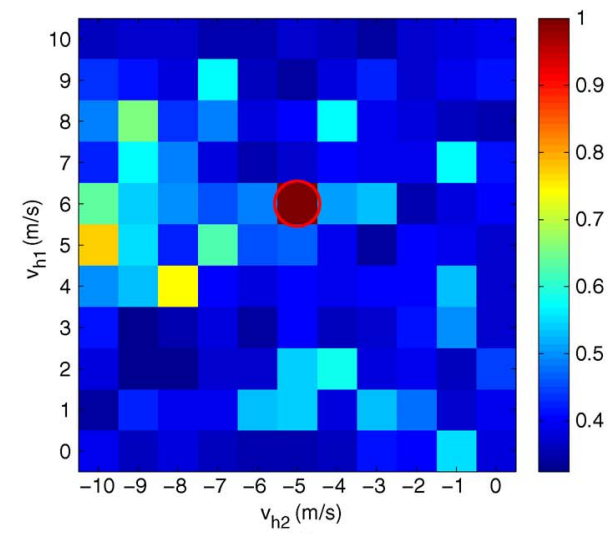

(c)

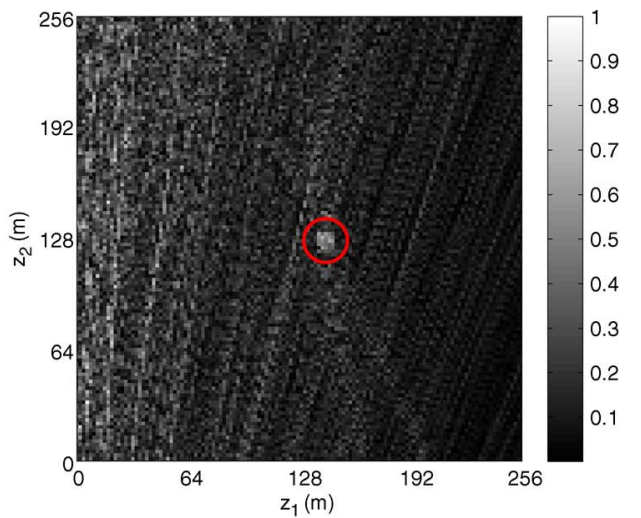

(b)

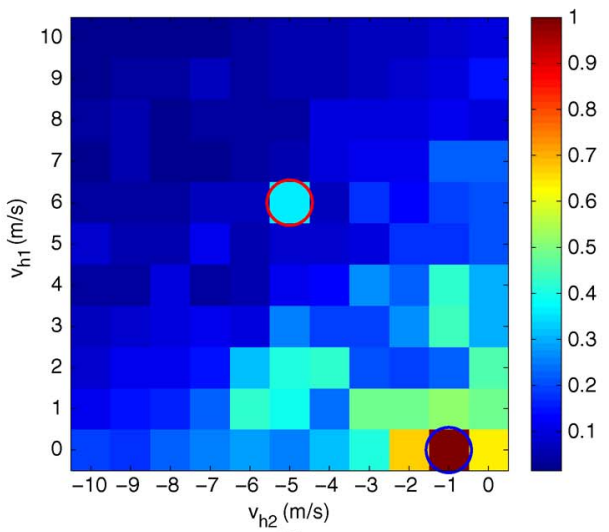

(d)

Fig. 12. Simulation results at low SCNR $(12 \mathrm{~dB})$. (a) Original scene. (b) Moving target reflectivity image reconstructed with the estimated velocity $\tilde{\boldsymbol{v}}_{\mathbf{x}}^{c}=$ $[6,-5] \mathrm{m} / \mathrm{s}$. The red circle indicates the true target position at time $t=0$. (c) Contrast and (d) gradient images with $\tilde{\boldsymbol{v}}_{\mathbf{x}}^{c}=[6,-5] \mathrm{m} / \mathrm{s}$ and $\tilde{\boldsymbol{v}}_{\mathbf{x}}^{g}=[0,-1] \mathrm{m} / \mathrm{s}$. The red circles in (c) and (d) indicate the true velocities, whereas the blue circle indicates the estimated velocity.

TABLE II

Simulation Parameters USED IN Four DifFERENT CASES

\begin{tabular}{l|lll}
\hline Cases & $f_{0}(\mathrm{MHz})$ & $L_{\phi}(\mathrm{s})$ & $L_{s}(\mathrm{~m})$ \\
\hline 1 & 800 & 0.0107 & $5.5 \mathrm{e} 3$ \\
2 & 80 & 0.0107 & $5.5 \mathrm{e} 3$ \\
3 & 800 & 0.1707 & $5.5 \mathrm{e} 3$ \\
4 & 800 & 0.0107 & $22 \mathrm{e} 3$ \\
\hline
\end{tabular}

reflectivity located at $[140,124,0] \mathrm{m}$, at $t=0$, moving with velocity $[6.2,-5.5] \mathrm{m} / \mathrm{s}$. Note that this position corresponds to the $(70,62)$ th pixel in the image.

We performed numerical simulations to demonstrate the effect of $f_{0}$, the carrier frequency of the transmitted waveform; $L_{\phi}$, the length of the support of $\phi(t)$; and $L_{s}$, the length of the aperture on the PSF. We reconstructed the PSF for the four cases listed in Table II.

Fig. 13(a)-(d) shows the reconstructed PSFs. For ease of comparison, the $X$ and $Y$ profiles for the four cases are shown together in Fig. 14(a) and (b). We also tabulated the 3-dB mainlobe width and the peak-to-sidelobe ratio (PSLR) of the $X$ and $Y$ profiles in Table III, where the 3-dB mainlobe width is used as a measure of resolution, and PSLR is used as a measure of the level of the sidelobes in the reconstructed PSFs. Note that, in the fourth case, the amplitude of the PSF smoothly attenuates, and there are no obvious peak sidelobes in the $X$ and $Y$ profiles of the PSF. We used the sidelobe level at the position corresponding to the peak sidelobe location in the first case as the peak sidelobe value in the fourth case.

As shown in Fig. 13(a) and (b), the quality of the reconstructed motion-compensated PSF degrades as the frequency of the transmitted waveform decreases from 800 to $80 \mathrm{MHz}$. We observe an obvious spreading of the mainlobes and an obvious increase in the level of the sidelobes in Fig. 14(a) and (b). This is also indicated by the increase in the 3-dB mainlobe width and the PSLR, as shown in Table III.

Comparing Fig. 13(c) with Fig. 13(a), we see that the quality of the PSF improves as the length of the support of the windowing function increases from 0.0107 to $0.1707 \mathrm{~s}$ due to a larger data collection manifold. Comparing the profiles of the PSFs in both cases, we see that the level of the sidelobes of the PSF decreases as the support of the windowing function gets longer, which is also indicated by the PSLR values in the two cases listed in Table III. The resolution in the X-direction and Y-direction also improves as compared to the first case as shown in Table III.

Comparing Fig. 13(d) with Fig. 13(a), we see that the increase in the synthetic aperture length $L_{s}$ results in an improvement in the reconstructed PSF as expected. We observe that there is a significant improvement in the sidelobes of the PSF, as shown in Fig. 14(a) and (b), which is also indicated by the decrease of the PSLR value from case (1) to case (4) in Table III. Furthermore, it is shown in Table III that the resolution in the $X$-direction and $Y$-direction improves. 


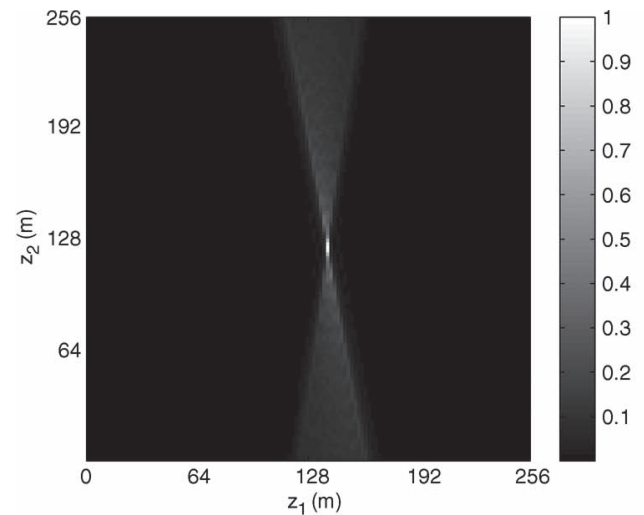

(a)

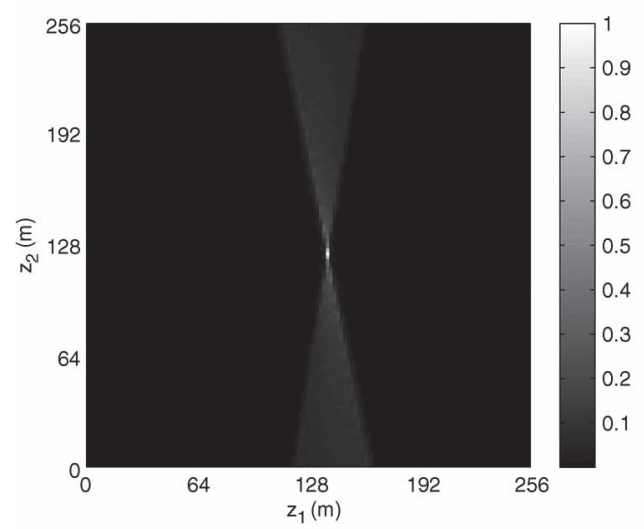

(c)

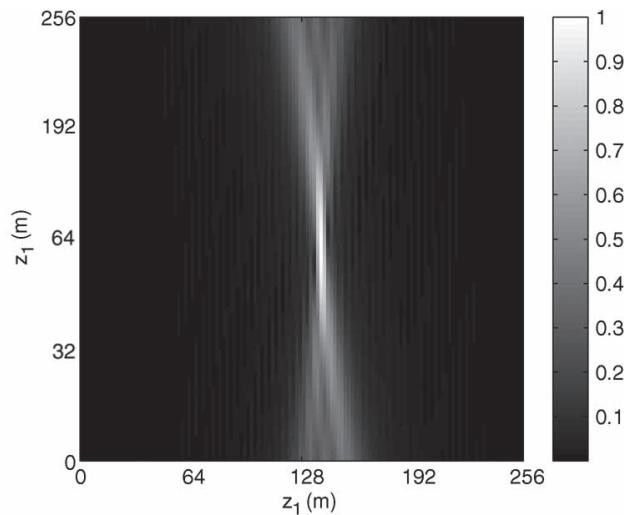

(b)

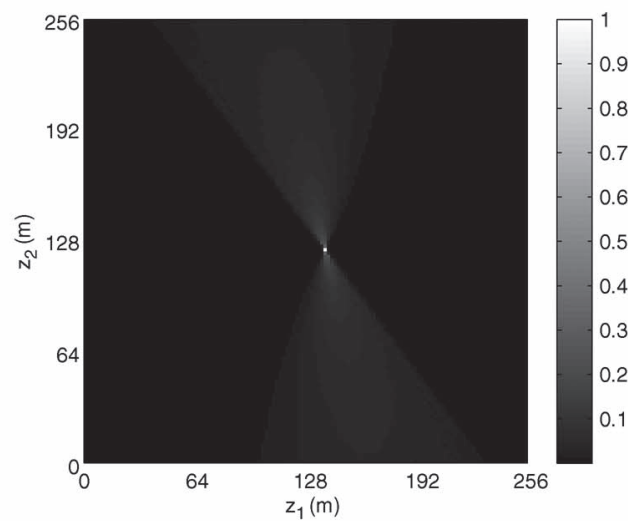

(d)

Fig. 13. Motion-compensated PSFs of the moving target imaging method reconstructed using different waveform parameters: (a) $f_{0}=800 \mathrm{MHz}, L_{\phi}=$ $0.0107 \mathrm{~s}$, and $L_{s}=5.5 e 3 \mathrm{~m}$; (b) $f_{0}=80 \mathrm{MHz}, L_{\phi}=0.0107 \mathrm{~s}$, and $L_{s}=5.5 e 3 \mathrm{~m}$; (c) $f_{0}=800 \mathrm{MHz}, L_{\phi}=0.1707 \mathrm{~s}$, and $L_{s}=5.5 e 3 \mathrm{~m}$; (d) $f_{0}=800 \mathrm{MHz}, L_{\phi}=0.0107 \mathrm{~s}$ and $L_{s}=22 e 3 \mathrm{~m}$.

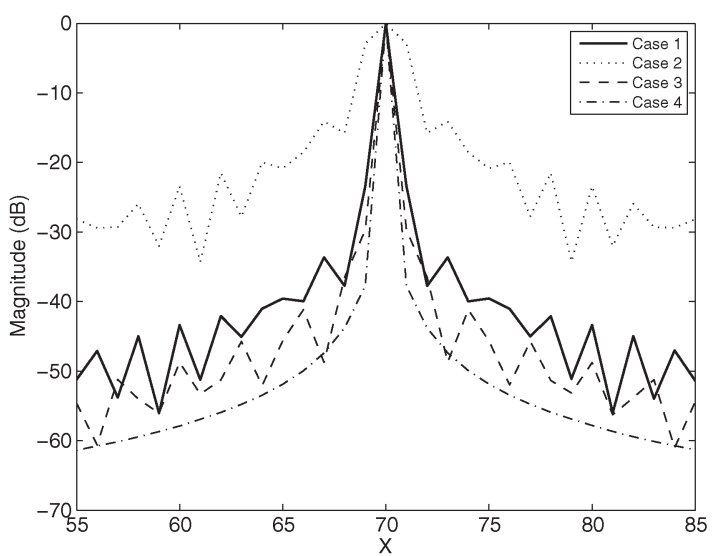

(a)

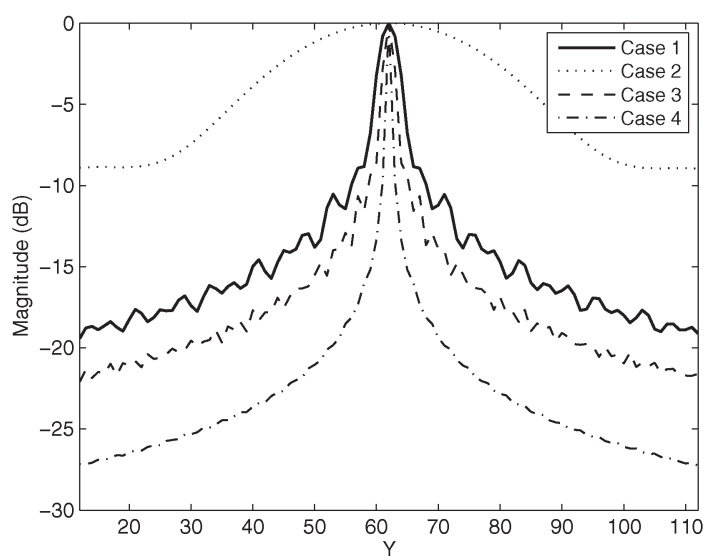

(b)

Fig. 14. (a) X and (b) Y profiles of the reconstructed PSFs shown in Fig. 13.

TABLE III

PSLR AND 3-dB MAINLOBE WIDTH OF THE RECONSTRUCTED PSFs FOR CASES 1-4

\begin{tabular}{l|cc|cc}
\hline & \multicolumn{2}{|c|}{ X direction } & \multicolumn{2}{c}{ Y direction } \\
Cases $\left(f_{0}, L_{\phi}, L_{s}\right)$ & 3dB Mainlobe width $(\mathrm{m})$ & PSLR $(\mathrm{dB})$ & 3dB Mainlobe width (m) & PSLR (dB) \\
\hline (1) $(800 \mathrm{MHz}, 0.0107 \mathrm{~s}, 5.5 \mathrm{e} 3 \mathrm{~m})$ & 0.50 & -33.6399 & 7.64 & -10.5361 \\
(2) $(80 \mathrm{MHz}, 0.0107 \mathrm{~s}, 5.5 \mathrm{e} 3 \mathrm{~m})$ & 4.05 & -14.1061 & 76.80 & -8.9015 \\
(3) $(800 \mathrm{MHz}, 0.1707 \mathrm{~s}, 5.5 \mathrm{e} 3 \mathrm{~m})$ & 0.41 & -41.1651 & 4.10 & -12.9181 \\
(4) $(800 \mathrm{MHz}, 0.0107 \mathrm{~s}, 22 \mathrm{e} 3 \mathrm{~m})$ & 0.32 & -47.1651 & 1.22 & -19.8301 \\
\hline
\end{tabular}


TABLE IV

TARget Parameters: Position, ReFlectivity, VElocity, AND SCNR

\begin{tabular}{l|cccc}
\hline Target No. & Center postion (pixel) & Velocity $(\mathrm{m} / \mathrm{s})$ & Reflectivity & SCNR (dB) \\
\hline 1 & $(27,21)^{\text {th }}$ & $(-10,15)$ & 10 & 26.49 \\
2 & $(111,91)^{\text {th }}$ & $(-10,15)$ & 3 & 16.03 \\
3 & $(27,31)^{\text {th }}$ & $(5,5)$ & 3 & 16.03 \\
4 & $(17,21)^{\text {th }}$ & $(-10,16)$ & 3 & 16.03 \\
5 & $(51,76)^{\text {th }}$ & $(15,-5)$ & 6 & 14.68 \\
\hline
\end{tabular}

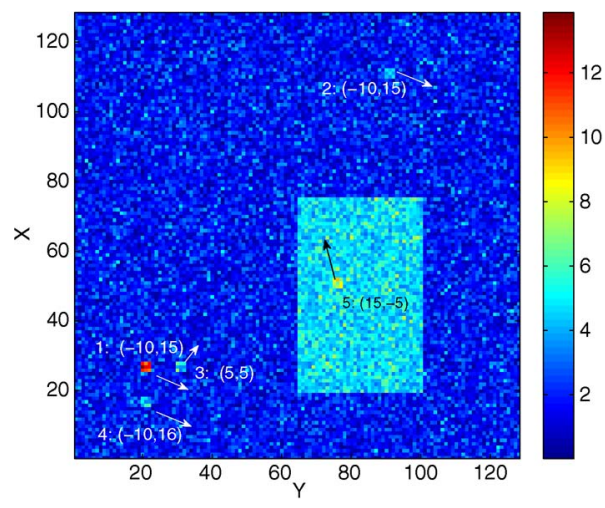

Fig. 15. Scene considered in the numerical simulations.

\section{Numerical Simulations for Multiple Moving Targets}

We considered a scene of size $[0,1100] \times[0,1100] \mathrm{m}^{2}$ with flat topography centered at $[11,11,0] \mathrm{km}$. The scene was discretized into $128 \times 128$ pixels, where $[0,0,0] \mathrm{m}$ and [1100, $1100,0] \mathrm{m}$ correspond to the pixels $(1,1)$ and $(128,128)$, respectively. We assumed that there were five moving targets present in the scene. Each moving target was simulated as a $3 \times 3$ pixel. We considered both uniform (homogeneous) clutter and structured (heterogeneous) clutter in the simulations. The former was simulated as an uncorrelated complex Gaussian random field with variance $\sigma_{c}^{2}=2$. The latter was simulated as a stationary relatively large extended target centered at the pixel $(48,83)$ of size $56 \times 36$ pixels with a reflectivity of 3 . The receiver noise was simulated as additive white Gaussian at the CNR level of $20 \mathrm{~dB}$.

Table IV lists the moving target parameters, including center position, velocity, reflectivity, and SCNR. Fig. 15 shows the scene with cluttered background and multiple moving targets along with their corresponding velocities. Note that the SCNRs in Table IV were calculated with respect to each moving target. The static target clutter was only taken into account in calculating the SCNR for the fifth target, which is superimposed on the static extended target. In fact, when we reconstruct an image of a particular moving target in a scene with multiple moving targets, other moving targets moving at different velocities contribute as an interference and can be viewed as clutter. However, we did not take this factor into account in the SCNR calculation. Note that this simulation setup includes the following cases: Two moving targets with very different SCNRs that are i) close in both position and velocity spaces, such as targets 1 and 4; ii) close in position, but far apart in velocity spaces, such as targets 1 and 3 ; iii) equal in velocity, but far apart in position spaces, such as Target 1 and 2; and iv) a moving target embedded in a stationary structured extended target/clutter.

We assumed that the transmitter and receiver were traversing a circular trajectory given by

$$
\gamma_{C}(\theta)=(11+11 \cos (\theta), 11+11 \sin (\theta), 6.5) \mathrm{km} .
$$

Let $\gamma_{T}(\theta)$ and $\gamma_{R}(\theta)$ denote the trajectories of the transmitter and the receiver. We set $\gamma_{T}(\theta)=\gamma_{C}(\theta)$ and $\gamma_{R}(\theta)=\gamma_{1}(\theta-$ $\pi / 4)$. Note that the variable $\theta$ in $\gamma_{C}$ is equal to $(v / R) t$, where $v$ is the speed of the receiver, and $R$ is the radius of the circular trajectory. We set the speed of the transmitter and receiver to $261 \mathrm{~m} / \mathrm{s}$.

We reconstructed $\tilde{\rho}_{\boldsymbol{v}_{h}}(\boldsymbol{z})$ images via the FBP method, as described in Section III and III-D, with $f_{0}=800 \mathrm{MHz}$ and $L_{\phi}=$ $0.0107 \mathrm{~s}$. The circular aperture was uniformly sampled into 2048 points, corresponding to an aperture sampling frequency $f_{s}=7.7340 \mathrm{~Hz}$. We assumed that the velocity of the targets is in the range of $[-20,20] \times[-20,20] \mathrm{m} / \mathrm{s}$ and discretized the entire velocity space into a $41 \times 41$ grid with a step size of $1 \mathrm{~m} / \mathrm{s}$.

Fig. 16(a) and (b) shows the contrast and gradient images formed, as described in Section III-D. We set the detection threshold at 1.5 times the mean value of the contrast image. The detection results based on the contrast image are shown in Fig. 16(c). We see that six velocities are detected, where the circles denote the correct detection, and the triangle denotes the false alarm. The results are favorable, since all five moving targets moving at four different velocities are all correctly detected.

We applied the same threshold as we determined from the contrast image to the gradient image to detect moving targets. The results show that only four velocities were detected, of which, two correspond to true moving target velocities, one corresponds to $(0,0)-\mathrm{m} / \mathrm{s}$ velocity (denoted by "star"), and one corresponds to a false alarm, as shown in Fig. 16(d). We reduced the threshold to allow the same number of detected velocities as in Fig. 16(c). The detection results are shown in Fig. 16(e), where two additional false alarms arise, as compared with Fig. 16(d).

Comparing Fig. 16(e) with Fig. 16(c), we see that the contrast-based velocity estimation outperforms the gradientbased velocity estimation. Only moving targets 1,2 , and 5 with velocities $[-10,15,0]$ and $[15,-5,0] \mathrm{m} / \mathrm{s}$ are correctly detected in the latter case, whereas all moving targets are detected in the former case.

Fig. 17 shows the reconstructed reflectivity images of the moving targets using the four correctly estimated velocities obtained using the contrast-based metric. These velocities are 


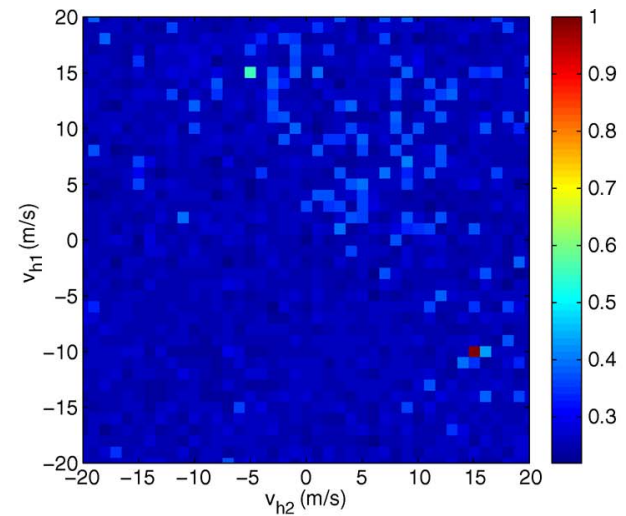

(a)

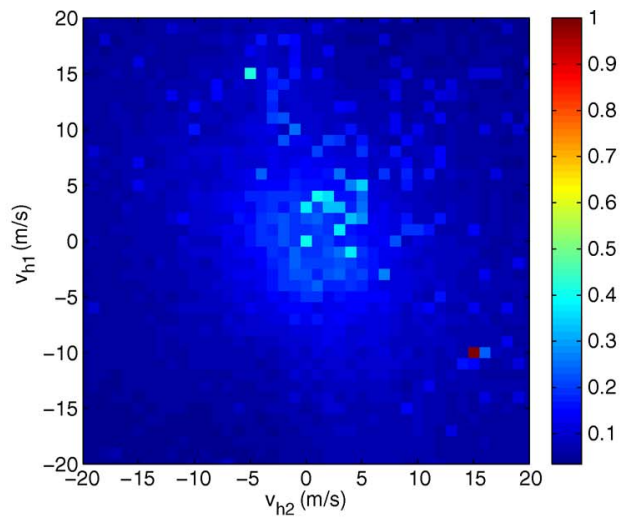

(b)

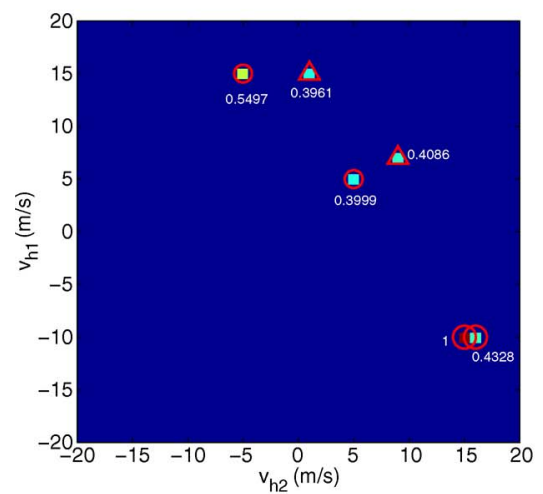

(c)

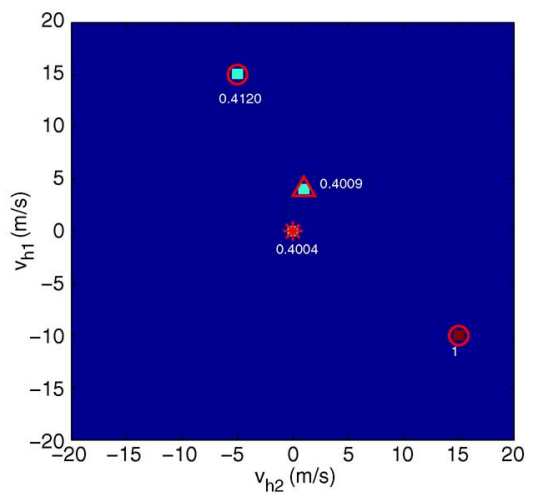

(d)

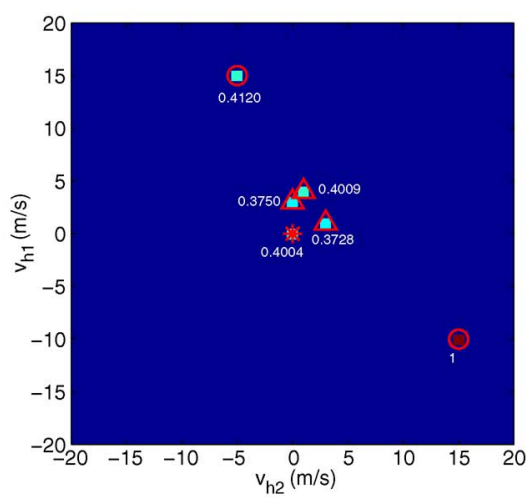

(e)

Fig. 16. (a) Contrast and (b) gradient images formed by the contrast and gradient of the images reconstructed with each hypothesized velocity. (c) Contrast and (d) gradient images after thresholding with the same threshold. (e) Thresholded gradient image with a lower threshold, as compared with that used in (d).

$[-10,15],[5,5],[-10,16]$, and $[15,-5] \mathrm{m} / \mathrm{s}$. Note that the moving targets are all well focused in the reconstructed images corresponding to the correct velocities.

Note that the moving targets 3 and 4 are missed in the gradient-based detection. This is mainly due to the interference caused by the unfocused and strong moving target 1 that is nearby and the unfocused and large extended stationary target that has the same reflectivity as targets 3 and 4, as shown in Fig. 17(b) and (c). On the other hand, the gradient-based approach can detect the stationary large extended target, whereas the image contrast measure fails, as shown in Fig. 16(c) and (e).

\section{Conclusion}

We considered the problem of SAR imaging of moving targets using ultranarrowband $\mathrm{CW}$ signals. We developed a received signal model for a moving scene and a novel forward model for image formation. Unlike the conventional wideband SAR forward model, the new forward model does not use startstop approximation. Instead, it exploits the temporal Doppler induced by the movement of antennas and moving targets for the image formation.

We developed an FBP-type method to reconstruct the reflectivity of a moving scene. The reflectivity reconstruction involves filtering and backprojecting the correlated signal onto the bistatic iso-Doppler contours for a range of hypothesized velocities. We use the image contrast and gradient optimization to estimate the velocity of moving targets from the stack of reflectivity images corresponding to a range of hypothesized velocities. The numerical simulations show that the image contrast outperforms the image gradient in the velocity estimation under low SCNRs. We analyzed the resolution of reconstructed reflectivity images via the PSF of the imaging operator. Our analysis shows that the reflectivity resolution is determined by the temporal duration and the carrier frequency of the transmitted waveforms. These findings are consistent with the Doppler ambiguity theory of CWs.

The stationary scene reconstruction method using ultranarrowband SAR that we reported in [33] can be implemented with the computational complexity of fast backprojection algorithms [32], [42]-[44]. As compared with [33], the computational complexity of our moving target image reconstruction and velocity estimation method increases roughly by a factor of $M^{2}$ if the velocity space is discretized into $M \times M$ points. Nonetheless, since $M^{2}$ is typically much smaller than the dimensions of the reflectivity image, the method can be implemented efficiently by using fast backprojection algorithms [42], [43], or fast Fourier integral operator computation methods [32], [44], and by utilizing parallel processing on graphics processing units [45], [46].

There are many important problems that may arise in application of our method to practical scenarios. Some of these problems relate to low signal-to-noise ratio or signal-to-clutter ratio, nonlinear target motion, the angular dependence of scene reflectivity due to wide bistatic apertures, and sensitivity of our method to imprecise antenna trajectory information. 


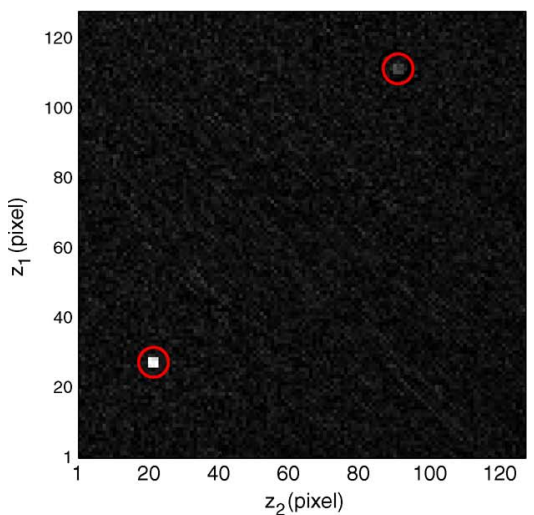

(a)

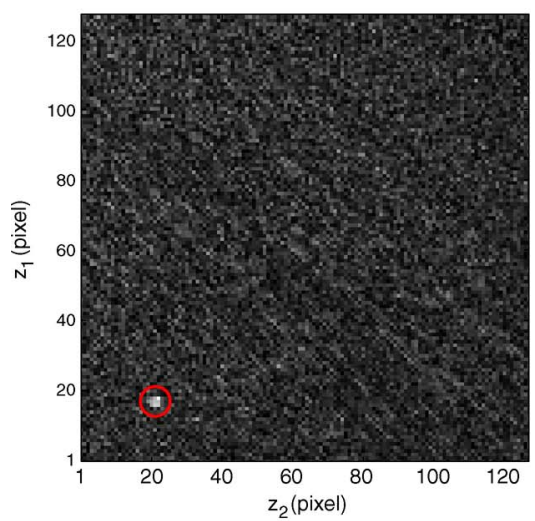

(c)

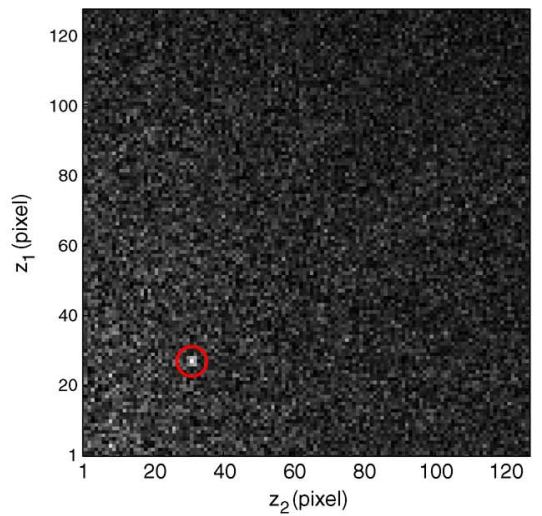

(b)

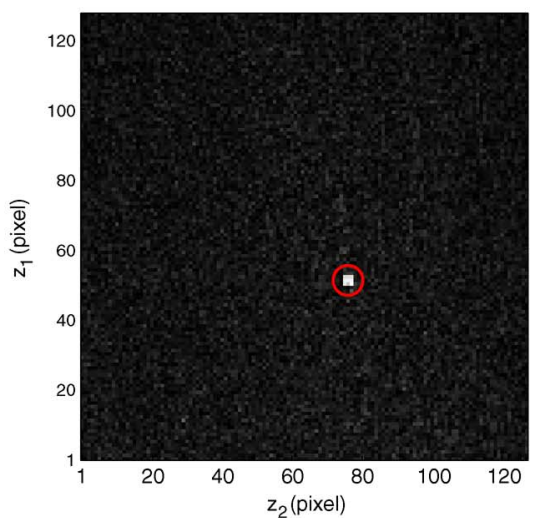

(d)

Fig. 17. Reconstructed images with the estimated target velocities: (a) $\tilde{\mathbf{v}}_{1,2}=[-10,15,0] \mathrm{m} / \mathrm{s} ;$ (b) $\tilde{\mathbf{v}}_{3}=[5,5,0] \mathrm{m} / \mathrm{s} ;$ (c) $\tilde{\mathbf{v}}_{4}=[-10,16,0] \mathrm{m} / \mathrm{s}$; and (d) $\tilde{\mathbf{v}}_{5}=[15,-5,0] \mathrm{m} / \mathrm{s}$. Note that the subscript corresponds to the number of the target shown in Fig. 15 and listed in Table IV.

Although our imaging scheme was developed in a deterministic setting, it is also applicable when the measurements are corrupted by additive white Gaussian noise [47]. When a priori information for the scene to be reconstructed is available and the additive noise and clutter are colored, the FBP-type inversion method presented in this paper can be extended, as described in [48]. The angular dependence of scene reflectivity can be addressed by a number of approaches that include incoherent superposition of images reconstructed using data from subapertures [49] or by using full polarimetric measurements [50]. While our moving target imaging method primarily considers linear target motion, our forward model and inversion method can be extended to include higher order kinetic parameters. Another important issue is the sensitivity or robustness of our target position and velocity estimation to imprecise antenna trajectories and ground topography information. We leave the investigation of these problems to ultranarrowband CW SAR imaging for the future.

Finally, while our primarily interest is in radar imaging, our method is also applicable to other similar imaging problems such as those that may arise in acoustics.

\section{REFERENCES}

[1] S. Werness, W. Carrara, L. Joyce, and D. Franczak, "Moving target imaging for SAR data," IEEE Trans. Aerosp. Electron. Syst., vol. 26, no. 1, pp. 57-67, Jan. 1990.

[2] H. Yang and M. Soumekh, "Blind-velocity SAR/ISAR imaging of a moving target in a stationary background," IEEE Trans. Image Process., vol. 2, no. 1, pp. 80-95, Jan. 1993.
[3] S. Barbarossa, "Detection and imaging of moving objects with synthetic aperture radar-Part 1: Optimal detection and parameter estimation theory," Proc. Inst. Elect. Eng. F, vol. 139, no. 1, pp. 79-88, Feb. 1992.

[4] S. Barbarossa, "Detection and imaging of moving objects with synthetic aperture radar-Part 2: Joint time-frequency analysis by Wigner-Ville distribution," Proc. Inst. Elect. Eng. F, vol. 139, no. 1, pp. 89-97, Feb. 1992.

[5] B. Friedlander and B. Porat, "VSAR: A high resolution radar system for detection of moving targets," Proc. Inst. Elect. Eng.-Radar, Sonar Navig., vol. 144, no. 4, pp. 205-218, Aug. 1997.

[6] C. V. Jakowatz, J. D. E. Wahl, and P. H. Eichel, "Refocus of constant velocity moving targets in synthetic aperture radar imagery," in Proc. SPIE Conf. Algorithms Synthetic Aperture Radar Imagery V, 1998, vol. 3370, pp. $85-95$.

[7] R. P. Perry, R. C. Dipietro, and R. L. Fante, "SAR imaging of moving targets," IEEE Trans. Aerosp. Electron. Syst., vol. 35, no. 1, pp. 188-200, Jan. 1999.

[8] Y. Ding and D. C. Munson, "Time-frequency methods in SAR imaging of moving targets," in Proc. IEEE Int. Conf. Acoust., Speech Signal Process., Orlando, FL, USA, May 2002, pp. III-2881-III-2884.

[9] J. R. Fienup, "Detection moving targets in SAR imagery by focusing," IEEE Trans. Aerosp. Electron. Syst., vol. 37, no. 3, pp. 794-809, Jul. 2001.

[10] J. K. Jao, "Theory of synthetic aperture radar imaging of a moving target," IEEE Trans. Geosci. Remote Sens., vol. 39, no. 9, pp. 1984-1992, Sep. 2001.

[11] M. Soumekh, "Moving target detection and imaging using an $\mathrm{x}$ band along-track monopulse SAR," IEEE Trans. Aerosp. Electron. Syst., vol. 38, no. 1, pp. 315-333, Jan. 2002.

[12] V. C. Chen, "Radar detection of multiple moving targets in clutter using time-frequency radon transform," in Proc. SPIE Conf. Signal Data Process. Small Targets, 2002, vol. 4728, pp. 48-59.

[13] M. Kirscht, "Detection and imaging of arbitrarily moving targets with single-channel SAR," Proc. Inst. Elect. Eng.-Radar Sonar Navig., vol. 150, no. 1, pp. 7-11, Feb. 2003.

[14] M. I. Pettersson, "Detection of moving targets in wideband SAR," IEEE Trans. Aerosp. Electron. Syst., vol. 40, no. 3, pp. 780-796, Jul. 2004. 
[15] M. J. Minardi, L. A. Gorham, and E. G. Zelnio, "Ground moving target detection and tracking based on generalized SAR processing and change detection," in Proc. SPIE Defense, Secur. Sens., Bellingham, WA, USA, Apr. 2005, vol. 5808, pp. 156-165.

[16] D. Cerutti-Maori, J. Klare, A. R. Brenner, and J. H. G. Ender, "Wide area traffic monitoring with the SAR/GMTI system PAMIR," IEEE Trans. Geosci. Remote Sens., vol. 46, no. 10, pp. 3019-3030, Oct. 2008.

[17] D. E. Hack and M. A. Saville, "Analysis of SAR moving grid processing for focusing and detection of ground moving targets," in Proc. SPIE Defense, Secur. Sens., Orlando, FL, USA, Apr. 2011, vol. 8051, p. 80510 S.

[18] R. W. Deming, "Along-track interferometry for simultaneous SAR and GMTI: Application to Gotcha challenge data," in Proc. SPIE Conf. Algorithms Synthetic Aperture Radar Imagery XVIII, 2011, vol. 8051, pp. P1-P18.

[19] D. Vu, B. Guo, L. Zhou, and J. Li, "Ground moving target indication via multi-channel airborne SAR," in Proc. SPIE Conf. Algorithms Synthetic Aperture Radar Imag. XVIII, 2011, vol. 8051, pp. Q1-Q12.

[20] S. Zhu, G. Liao, Y. Qu, Z. Zhou, and X. Liu, "Ground moving targets imaging algorithm for synthetic aperture radar," IEEE Trans. Geosci. Remote Sens., vol. 49, no. 1, pp. 462-477, Jan. 2011.

[21] M. Martorella, F. Berizzi, E. Giusti, and A. Bacci, "Refocussing of moving targets in SAR images based on inversion mapping and ISAR processing," in Proc. IEEE Int. Radar Conf., Kansas City, MO, USA, 2011, pp. 68-72.

[22] L. Wang and B. Yazici, "Bistatic synthetic aperture radar imaging using ultra-narrowband continuous waveforms," IEEE Trans. Image Process., vol. 21, no. 8, pp. 3673-3686, Aug. 2012.

[23] C. E. Yarman, L. Wang, and B. Yazıc1, "Doppler synthetic aperture hitchhiker imaging," Inverse Probl., vol. 26, no. 6, pp. 065006-1-065006-26, Jun. 2010.

[24] L. Wang, C. E. Yarman, and B. Yazici, "Doppler hitchhiker: A novel passive synthetic aperture radar using ultranarrowband sources of opportunity," IEEE Trans. Geosci. Remote Sens., vol. 49, no. 10, pp. 35213537, Oct. 2011.

[25] C. V. Jakowatz, J. D. E. Wahl, P. H. Eichel, D. C. Ghiglia, and P. A. Thompson, Spotlight-Mode Synthetic Aperture Radar: A Signal Processing Approach. Norwell, MA, USA: Kluwer, 1996.

[26] W. C. Carrara, R. G. Goodman, and R. M. Majewski, Spotlight Synthetic Aperture Radar: Signal Processing Algorithms. Boston, MA, USA: Artech House, 1995.

[27] A. Meta, "Signal processing of FMCW synthetic aperture radar data," Ph.D. dissertation, Delft Univ. Technol., Delft, The Netherlands, 2006.

[28] Y. Liang, H. Wang, M. Xing, and Z. Bao, "Slow ground moving target parameter estimation and imaging in FMCW SAR," Syst. Eng. Electron. (China), vol. 33, no. 5, pp. 1001-1006, May 2011.

[29] M. Stuff, M. Biancalana, G. Arnold, and J. Garbarino, "Imaging moving objects in 3D from single aperture synthetic aperture data," in Proc. IEEE Radar Conf., 2004, pp. 94-98.

[30] F. Zhou, R. Wu, M. Xing, and Z. Bao, "Approach for single channel SAR ground moving target imaging and motion parameter estimation," IET Radar Sonar Navig., vol. 1, no. 1, pp. 59-66, Feb. 2007.

[31] L. Borcea, T. Callaghan, and G. Papanicolaou, "Synthetic aperture radar imaging with motion estimation and autofocus," Inverse Probl., vol. 28, no. 4, pp. 045006-1-045006-31, Apr. 2012.

[32] L. Demanet, M. Ferrara, N. Maxwell, J. Poulson, and L. Ying, "A butterfly algorithm for synthetic aperture radar imaging," SIAM J. Imag. Sci., vol. 5, no. 1, pp. 203-243, Jan. 2012.

[33] L. Wang and B. Yazıc1, "Bistatic synthetic aperture radar imaging of moving targets using ultra-narrowband continuous waveforms," SIAM J. Imag. Sci., 2013, submitted for publication.

[34] M. Soumekh, Fourier Array Imaging. Upper Saddle River, NJ, USA: Prentice-Hall, 1994.

[35] L. Wang, C. E. Yarman, and B. Yazıc1, "Theory of passive synthetic aperture imaging," in Part II: Remote Sensing, Excursions in Harmonic Analysis. Boston, MA, USA: Springer-Birkhäuser, 2013, ser. Applied and Numerical Harmonic Analysis (ANHA) Book Series.

[36] B. Yazıcı and V. Krishnan, Microlocal Analysis in Imaging Tutorial, Mar. 2010. [Online]. Available: http://www.ecse.rpi.edu/ yazici/ ICASSPTutorial/

[37] F. Treves, Introduction to Pseudodifferential and Fourier Integral Operators. New York, NY, USA: Plenum, 1980.

[38] M. Martorella, F. Berizzi, and B. Haywood, "Contrast maximisation based technique for 2-D ISAR autofocusing," Proc. Inst. Elect. Eng.-Radar Sonar Navig., vol. 152, no. 4, pp. 253-262, Aug. 2005.

[39] M. Skolnik, Radar Handbook, 2nd ed. New York, NY, USA: McGrawHill, 1990.

[40] N. Levanon and E. Mozeson, Radar Signals. Hoboken, NJ, USA: WileyIEEE, 2004.
[41] O. C. and Q. S., Understanding Synthetic Aperture Radar Images. Raleigh, NC, USA: Scitech Publ., 2004.

[42] S. Nilsson, "Application of fast backprojection techniques for some inverse problems of integral geometry (Linköping Studies in Science and Technology)," Ph.D. dissertation, Dept. Math., Linköping Univ., Linköping, Sweden, 1997, Dissertation No. 499.

[43] L. Ulander, H. Hellsten, and G. Stenström, "Synthetic-aperture radar processing using fast factorized back-projection," IEEE Trans. Aerosp. Electron. Syst., vol. 39, no. 3, pp. 760-776, Jul. 2003.

[44] E. Candés, L. Demanet, and L. Ying, "Fast computation of Fourier integral operators," SIAM J. Sci. Comput., vol. 29, no. 6, pp. 2463-2493, Oct. 2007.

[45] A. Tasora, D. Negrut, and M. Anitescu, "GPU-based parallel computing for the simulation of complex multibody systems with unilateral and bilateral constraints: An overview," in Computational Methods in Applied Sciences: Multibody Dynamics. New York, NY, USA: Springer-Verlag, 2011, pp. 283-307.

[46] A. Capozzoli, C. Curcio, and A. Liseno, "GPU-based $\omega$-k tomographic processing by 1D non-uniform FFTs," Progr. Electromagn. Res. M (PIER-M), vol. 23, pp. 279-298, 2012.

[47] K. Voccola, B. Yazici, M. Cheney, and M. Ferrara, "On the relationship between the generalized likelihood ratio test and backprojection for synthetic aperture radar imaging," in Proc. SPIE Defense Secur. Conf., Orlando, FL, USA, Apr. 2009, pp. 73350I-1-73350I-10.

[48] B. Yazici, M. Cheney, and C. E. Yarman, "Synthetic-aperture inversion in the presence of noise and clutter," Inverse Probl., vol. 22, no. 5, pp. 17051729, Oct. 2006.

[49] V. Krishnan, J. Swoboda, C. E. Yarman, and B. Yazıcı, "Multi-static synthetic aperture radar image formation," IEEE Trans. Image Process., vol. 19 , no. 5, pp. 1290-1306, May 2010.

[50] K. Voccola, M. Cheney, and B. Yazici, "Polarimetric synthetic-aperture inversion for extended targets in clutter," Inverse Probl., vol. 29, no. 5, pp. 054003-1-054003-25, May 2013.

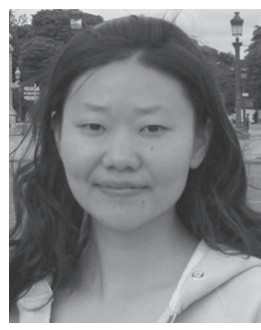

Ling Wang (M'07) received the B.S. degree in electrical engineering and the M.S. and Ph.D. degrees in information acquirement and processing from the Nanjing University of Aeronautics and Astronautics, Nanjing, China, in 2000, 2003, and 2006, respectively.

Since 2003, she has been with the Nanjing University of Aeronautics and Astronautics, where she is currently an Associate Professor with the Department of Information and Communication Engineering. From February 2008 to May 2009, she was a Postdoctoral Research Associate with the Department of Mathematical Sciences and the Department of Electrical, Computer, and Systems Engineering, Rensselaer Polytechnic Institute, Troy, NY, USA. Her current research interests include inverse scattering, wave-based imaging, radar imaging, and passive imaging.

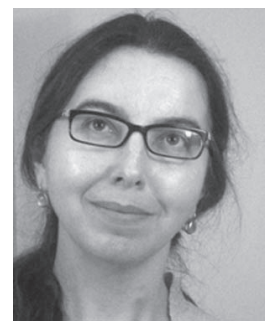

Birsen Yazici (SM'05) received the B.S. degree in electrical engineering and mathematics from Bogazici University, Istanbul, Turkey, in 1988 and the M.S. and Ph.D. degrees in mathematics and electrical engineering from Purdue University, West Lafayette, IN, USA, in 1990 and 1994, respectively.

From September 1994 to 2000, she was a Research Engineer with the General Electric Company Global Research Center, Schenectady, NY, USA. During her tenure in industry, she worked on radar, transportation, and industrial and medical imaging systems. From 2001 to 2003, she was an Assistant Professor with the Department of Electrical and Computer Engineering, Drexel University, Philadelphia, PA, USA. Since 2003, she has been with Rensselaer Polytechnic Institute, Troy, NY, where she is currently a Professor with the Departments of Electrical, Computer, and Systems Engineering and Biomedical Engineering. She holds eleven U.S. patents. Her current research interests include statistical signal processing, inverse problems in imaging, image reconstruction, biomedical optics, radar, and X-ray imaging.

Dr. Yazici's work on industrial systems received the second Best Paper Award from the IEEE TRANSACTIONS ON INDUSTRIAL APPLICATIONS in 1997. She was the recipient of the Rensselaer Polytechnic Institute School of Engineering Research Excellence Award in 2007 and 2013. She served as an Associate Editor for IEEE TRANSACTIONS ON IMAGE PROCESSING from 2007-2012. She currently serves as an Associate Editor for the SIAM Journal on Imaging Science. 